\title{
Extended near hexagons and line systems
}

\author{
Hans Cuypers \\ Department of Mathematics \\ Eindhoven University of Technology \\ P.O. Box 513, 5600 MB Eindhoven \\ The Netherlands \\ email: hansc@win.tue.nl
}

October 2, 2002

\begin{abstract}
In this paper we study extended near hexagons, and classify a class of line systems in which two lines are either perpendicular, or make an angle $\alpha$ with $\cos \alpha= \pm 1 / 3$. Among the examples we encounter a set of 2300 lines in $\mathbb{R}^{23}$ related to the second Conway group $\mathrm{Co}_{2}$ and a set of 2048 lines in $\mathbb{R}^{24}$ related to the group $2^{1+11}: \mathrm{M}_{24}$. The other line systems under consideration in this paper are all subsystems of these.
\end{abstract}

MSC classification: 51E30, 05B99, 51E12, 20D8

Keywords: Extended near hexagons, line systems, spherical designs.

\section{Introduction}

In [34], Shult and Yanushka studied a class of line systems in $\mathbb{R}^{n}$, equipped with the standard inner product $(\cdot \mid \cdot)$, in which any pair of distinct lines is either perpendicular or makes an angle $\alpha$ with $\cos \alpha= \pm 1 / 3$. A nice and simple example of such a line system is the set of 4 lines in $\mathbb{R}^{3}$ passing through the vertices of a regular tetrahedron centered at the origin. Other examples considered by Shult and Yanushka include a system of 2300 lines in $\mathbb{R}^{23}$ related to the Leech lattice and the second Conway group $\mathrm{Co}_{2}$ and a system of 2048 lines in $\mathbb{R}^{24}$ related to the binary Golay code and the group $2^{1+11}: \mathrm{M}_{24}$. These line systems are all tetrahedrally closed, i.e., given three lines of the system passing through three of the four vertices of a regular tetrahedron centered at the origin, then also the line passing through the fourth vertex of the tetrahedron is present in the line system. 
The study of tetrahedrally closed line systems led Shult and Yanushka to the notion of a near polygon. Consider a tetrahedrally closed line system $\mathcal{L}$, and denote by $\Sigma$ the set of norm 3 vectors on the lines in $\mathcal{L}$. (Here the norm of a vector $v$ equals $(v \mid v)$.) Let $v \in \Sigma$. Then by $\Sigma_{i}(v)$ we denote the set of vectors in $\Sigma$ having inner product $i$ with $v$. Here the relevant values of $i$ are of course $\pm 3, \pm 1$ and 0 . For each pair of vectors $u$ and $w$ in $\Sigma_{-1}(v)$ that have inner product -1 , there is a unique tetrahedron with the three vectors $v, u$ and $w$ as vertices. The fourth vertex $-(v+u+w)$ of this tetrahedron is then also a vector in $\Sigma_{-1}(v)$. If one takes the vectors in $\Sigma_{-1}(v)$ as points, and the triples of vectors in $\Sigma_{-1}(v)$ that together with $v$ are the four vertices of a regular tetrahedron on $v$ as lines, then under some additional conditions on connectedness this point-line geometry satisfies the following axiom:

(NP) given a line $l$ and point $p$, there is a unique point on $l$ closest to $p$;

which is the defining axiom of a near polygon.

The additional connectedness conditions forcing NP are:

(C) Every pair of vertices $v$ and $w$ with inner product -1 is contained in a tetrahedron.

Suppose $T$ is a tetrahedron on $v$, whose vertices are in $\Sigma$, and $x$ is an element of $\Sigma_{-1}(v)$, having inner product 0 or 1 with the three vertices of $T$ distinct from $v$. Then there is a vector $y \in \Sigma_{-1}(v) \cap \Sigma_{-1}(x)$ having inner product -1 with at least 1 of the three vertices of $T$ distinct from $v$.

This is the revised version by Neumaier (see [29]) of Shult and Yanushka's condition to force connectedness of the local point-line geometry.

Indeed, if $T$ is a tetrahedron on $v$ whose three other vertices are in in $\Sigma_{-1}(v)$, then for every vertex $x \in \Sigma_{-1}(v)$ we have one of the following possibilities. The vector $x$ is a vertex of $T$, or it has inner product -1 with exactly one vertex of $w$ of $T$ distinct from $v$ and 1 with the remaining two vertices, or it is perpendicular to two vertices in $T$ and has inner product 1 with the third vertex in $T$ distinct from $v$. In the second case, the vertices $v, x$ and $w$ are again in a tetrahedron.

Notice that two vertices $x, y \in \Sigma_{-1}(v)$ are at distance 1,2 or 3 inside the geometry induced on $\Sigma_{-1}(v)$ if and only if their inner product equals $-1,1$ or 0 respectively.

So, if the line system is tetrahedrally closed and satisfies condition (C), then the vectors in $\Sigma$ together with the set of tetrahedra with vertices in $\Sigma$ is an extended near hexagon in the terminology of Cameron et al. [14]. This extended near hexagon is a 2 -fold cover of the extended near hexagon that we can define on the line system $\mathcal{L}$, where the blocks or circles correspond to the sets of 4 lines in $\mathcal{L}$ through the vertices 


\section{EXTENDED NEAR HEXAGONS AND LINE SYSTEMS}

of the tetrahedra. The point graph of the extended near hexagon on $\Sigma$ is a graph which is locally the near hexagon; the point graph of the extended near hexagon defined on $\mathcal{L}$, however, is locally the distance 1-or-2 graph of the near hexagon. So the extended near hexagon on the lines in $\mathcal{L}$ satisfies the following condition:

$(*)\{x, y, z\}$ is a triangle in the point graph not contained in a circle, if and only if the distance between $y$ and $z$ in the local near hexagon at $x$ is 2 .

To ensure connectivity of the extended near hexagons, one can restrict attention to line systems that are indecomposable. That means, there is no non trivial partition of the set $\mathcal{L}$ in which any two lines from distinct parts of the partition are perpendicular.

The theory of near polygons has been developed by the work of several authors, see for example $[7,8,13]$, and even led to the full classification of all near hexagons with 3 points per line in which any two points at distance $\leq 2$ are in some quad, a near hexagon in which a point is always collinear to some point of a line, see [8].

The study of extended near polygons started with the work of Buekenhout and Hubaut [11]. Considerable work has been done on extensions of near polygons, in particular of generalized polygons, see $[13,28,36,37,38]$. For an overview of some of these results, the reader is referred to the chapter by Buekenhout and Pasini in the Handbook of Incidence Geometry [12]. However, since the pioneering work of Shult and Yanushka [34], and the article of Neumaier [29], as far as we know, no progress has been made on the study of the indecomposable tetrahedrally closed line systems.

In this paper we study both extensions of near hexagons and their connections with tetrahedrally closed line systems. We call a line system in which two lines either are perpendicular or make an angle $\alpha$ with $\cos \alpha= \pm 1 / 3$ regular, if and only if it is indecomposable, tetrahedrally closed, for each vector $v$ of norm 3 on one of the lines in $\mathcal{L}$ condition $(\mathrm{C})$ is satisfied, and the near hexagon on $\Sigma_{-1}(v)$ is regular. Our main result on line systems reads as follows.

Theorem 1.1 Let $\mathcal{L}$ be a line system in $\mathbb{R}^{n}$, such that any pair of lines is either perpendicular or makes an angle $\alpha$ with $\cos \alpha= \pm 1 / 3$. If $\mathcal{L}$ is regular, then it is isomorphic to one of the following systems:

i. the system of 10 lines in $\mathbb{R}^{5}$ related to $2 \times \mathrm{PSp}_{4}(2)$;

ii. the system of 16 lines in $\mathbb{R}^{6}$ related to $2^{1+4}: \operatorname{Sp}_{4}(2)$;

iii. the system of 28 lines in $\mathbb{R}^{7}$ related to $2 \times \operatorname{PSp}_{6}(2)$;

$i v$. the system of 40 lines in $\mathbb{R}^{10}$ related to $2 \times \operatorname{PSU}_{4}(2)$; 
$v$. the system of 120 lines in $\mathbb{R}^{15}$ related to $2 \times \mathrm{PSp}_{6}(2)$;

vi. the system of 128 lines in $\mathbb{R}^{16}$ related to $2^{1+7}: \mathrm{G}_{2}(2)$;

vii. the system of 256 lines in $\mathbb{R}^{16}$ related to $2^{1+8}: \operatorname{Sp}_{6}(2)$;

viii. the system of 2048 lines in $\mathbb{R}^{24}$ related to $2^{1+11}: \mathrm{M}_{24}$;

$i x$. the system of 2300 lines in $\mathbb{R}^{23}$ related to $2 \times \mathrm{Co}_{2}$.

In fact, all these line systems, except for the sixth one, have been described by Shult and Yanushka [34], who also gave some partial uniqueness results. A description of these systems will also be given in Section 3 of this paper.

Certainly the most interesting example is the set of 2300 lines in $\mathbb{R}^{23}$ related to the second Conway group $\mathrm{Co}_{2}$. It contains all the other line systems, except for the one on 2048 lines related to $2^{1+11}: \mathrm{M}_{24}$.

Our proof of Theorem 1.1 starts with the extended near hexagon defined on the line system. As observed above, the point graph of this geometry is a connected graph which is locally the distance 1-or-2 graph of a regular near hexagon. Such geometries are classified in the following result, which is the main theorem of this paper.

Main Theorem 1.2 Let $\Gamma$ be an extended near hexagon with regular local near hexagons having 3 points per line. If $\Gamma$ satisfies $(*)$, then $\Gamma$ is isomorphic to one of the following:

i. the extended generalized quadrangle on 10 points related to $\mathrm{PSp}_{4}(2)$;

ii. the extended generalized quadrangle on 16 points related to $2^{4}: \mathrm{Sp}_{4}(2)$;

iii. the extended generalized quadrangle on 28 points related to $\operatorname{PSp}_{6}(2)$;

$i v$. the extended near hexagon on 40 points related to $\mathrm{PSU}_{4}(2)$;

$v$. the extended generalized hexagon on 120 points related to $\operatorname{PSp}_{6}(2)$;

vi. the extended generalized hexagon on 128 points related to the group $2^{7}: \mathrm{G}_{2}(2)$;

vii. the extended near hexagon on 256 points related to $2^{8}: \operatorname{Sp}_{6}(2)$;

viii. the extended near hexagon on 2048 points related to $2^{11}: \mathrm{M}_{24}$;

ix. the extended near hexagon on 2300 points related to $\mathrm{Co}_{2}$. 
Theorem 1.1 is obtained as a corollary to the above result. As noticed above, any line system satisfying the conditions of Theorem 1.1 gives rise to an extended near hexagon as in Theorem 1.2. The set $\Sigma$ of norm 3 vectors of a line system carries also the structure of an extended near hexagon, and is a 2-fold cover of one of the examples in Theorem 1.2. Such covers can be determined, and the set $\Sigma$ can be recovered as a set of vectors obtained by projecting the points of the cover on an appropriate eigenspace of the adjacency matrix of its collinearity graph. This will be carried out in Section 6. .

The enrichment of an extended near hexagon, i.e., the geometry of points, pairs of points in a circle and circles (see [14]), is a diagram geometry with Buekenhout diagram

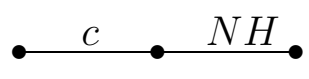

where $N H$ stands for the class of near hexagons. In particular, if the local near hexagon is a generalized hexagon we obtain a diagram geometry with diagram

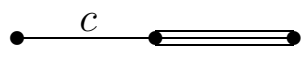

Moreover, as we will show in Section 4, the regular near hexagons containing quads yield geometries with diagram

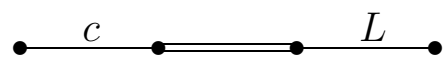

The geometries of Theorem 1.2 appear as subgeometries of the Baby monster geometry. The results of Theorem 1.2 have been used by Ivanov, Pasechnik and Sphectorov in their geometric characterization of the Baby Monster, see [27].

The enrichments of all the nine examples of extended near hexagons in the above theorem admit a flag-transitive automorphism group. Weiss [36, 37] has classified the extensions of classical generalized hexagon as diagram geometries admitting a flag-transitive group, and satisfying some additional conditions closely related to condition $(*)$ of Theorem 1.2. In [36], however, he missed the example on 120 points related to the group $\operatorname{PSp}_{6}(2)$. Weiss informed us that this example should have appeared in Lemma 4.5 of [36], as the group $G_{1,1,1}$. In [23], the author extended 
Weiss' result by classifying all flag-transitive extensions of buildings of type $\mathrm{G}_{2}$ or $\mathrm{C}_{3}^{*}$ under the same additional conditions. The $\mathrm{Co}_{2}$ extended near hexagon has also been characterized by Meixner [28] and by Yoshiara [38] as a flag-transitive extension of the $\mathrm{PSU}_{6}(2)$ dual polar space.

The case of a circular extension of a near hexagon of order 2 is a special case of an affine extension of a near hexagon. In $[4,5]$ it is shown that the examples (iv), (v) and (vii) are special cases of affine extensions of generalized hexagons of type $\mathrm{G}_{2}$ and dual polar spaces of type $\mathrm{C}_{3}$, respectively.

Extensions of regular line thick generalized hexagons whose point graph is locally the distance 1-or-3 graph of the local hexagon are investigated in [22]. There are just four examples of such extended hexagons; they are related to the almost simple groups $\mathrm{G}_{2}(2)$, HJ, Suz and $\mathrm{PSU}_{4}(3)$, see [22]. These examples are also characterized by Weiss $[36,37]$ and the author [23] under the assumption of a flag-transitive action of the automorphism group.

In the work of Weiss [36, 37], Yoshiara [38] and the author [23] also circular extensions of finite classical generalized hexagons and dual polar spaces of rank 3 are considered where the number of points per line is not restricted, but arbitrary. The methods we develop in Section 4 lead to the following theorem:

Theorem 1.3 Suppose $\Gamma$ is an extended near hexagon, such that for each point $p$ of $\Gamma$ the local near hexagon $\Gamma_{p}$ at $p$ is regular with classical parameters $\left(s, t_{2}, t\right)$ where $s \geq 2$. If $\Gamma$ satisfies $(*)$, then $s=2$. In particular, $\Gamma$ is one of the examples of the conclusion of Theorem 1.2.

Here, we say that a near hexagon has classical parameters $\left(s, t, t_{2}\right)$ if and only if either $t_{2}>0$ and $t=t_{2}\left(t_{2}+1\right)$ or $t_{2}=0$ and $t \in\left\{1, \sqrt[3]{s}, s, s^{3}\right\}$.

Acknowledgment. The author would like to thank John van Bon and Antonio Pasini for many valuable remarks and comments on the subject of this paper.

\section{Definition and notation}

In this section we recall some definitions and fix the notation used throughout this paper.

Let $\Gamma$ be the incidence structure $(\mathcal{P}, \mathcal{C})$ consisting of non empty sets $\mathcal{P}$ of points and $\mathcal{C}$ of circles (also called blocks), which are subsets of size at least 2 of $\mathcal{P}$. The point graph (or collinearity graph) of $\Gamma$ is the graph with vertex set $P$, and two points adjacent if and only if they are cocircular (also called collinear), i.e., there is a circle containing them. If $p$ and $q$ are points of $\mathcal{P}$, then we denote by $p \perp q$ that there is a circle containing them. For each subset $X$ of $\mathcal{P}$ we write $X^{\perp}$ for the set of all points $q$ with $q \perp x$ for all $x \in X$. For points $p \in \mathcal{P}$ we usually write $p^{\perp}$ instead of $\{p\}^{\perp}$. 
A subspace $X$ of $\Gamma$ is a subset of $\mathcal{P}$ such that each circle meeting it in at least 2 points is contained in $X$. A subspace $X$ is often tacitly identified with the incidence structure $(X,\{C \in \mathcal{C}|| C \cap X \mid \geq 2\})$. A proper subspace of $\Gamma$ meeting all circles non trivially is called a geometric hyperplane or, for short, hyperplane of $\Gamma$. A point in a geometric hyperplane is called deep, if all the lines on that point are contained in the geometric hyperplane.

We call $\Gamma$ a near polygon, if it satisfies the following condition:

(NP) given an element $C \in \mathcal{C}$ and $p \in \mathcal{P}$, then in the point graph of $\Gamma$ there is a unique point $q \in C$ at minimal distance from $p$.

Here distance is just the usual graph distance. A near polygon $\Gamma$ is called a near hexagon, if its point graph has diameter at most 3. It is called a generalized quadrangle, when its point graph has diameter at most 2 . In a near polygon circles are usually referred to as lines. If all lines of a near polygon have at least 3 points and two points at distance two have more than one common neighbor, then the geodesic closure of these two points is a subspace of the near polygon, carrying the structure of a generalized quadrangle. This result is known as Yanushka's Lemma, see [34]. Such subspaces are called quads.

A near polygon whose point graph is of diameter $d$, is called regular with parameters $\left(s, t_{2}, \ldots, t=t_{d}\right)$, if and only if all lines contain $s+1$ points, for every point $p$ there is a point $q$ at distance $d$ from $p$, and for each pair of points $(p, q)$ at distance $i$, where $2 \leq i \leq d$, there are exactly $t_{i}+1$ lines through $p$ containing a point at distance $i-1$ from $q$.

Consider an arbitrary incidence structure $\Gamma=(\mathcal{P}, \mathcal{L})$. Let $p$ be a point of $\Gamma$. Then by $\mathcal{P}_{p}$ we denote the set of points adjacent to $p$ in the point graph of $\Gamma$, and by $\mathcal{C}_{p}$ the set of all circles containing $p$. If all circles of $\Gamma$ contain at least 3 points, then we can consider the incidence system $\Gamma_{p}=\left(\mathcal{P}_{p},\left\{C-\{p\} \mid C \in \mathcal{C}_{p}\right\}\right)$, the residue of $\Gamma$ at $p$. We will call $\Gamma$ an extended near polygon, respectively, extended near hexagon, if and only if its point graph is connected all circles contain at least 3 points, and $\Gamma_{p}$ is a near polygon, respectively, near hexagon, for all points $p \in \mathcal{P}$.

If $\Gamma$ is an extended near polygon and $p$ is a point of $\Gamma$, then $\Gamma_{p}$ is also called the local near polygon at $p$.

The enrichment $\mathcal{E}(\Gamma)$ of $\Gamma$ is the incidence system $(\mathcal{P}, \mathcal{E}, \mathcal{C})$, where $\mathcal{E}$ is the set of edges of the point graph of $\Gamma$, and incidence is symmetrized inclusion. As was observed in [14], if $\Gamma$ is an extended near polygon, then this incidence system is a diagram geometry with Buekenhout diagram 


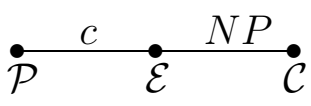

where $N P$ is a class of near polygons.

\section{Examples of line systems and extended near hexagons}

The examples of line systems and extensions of near hexagons appearing in the theorems of the introduction of this paper will briefly be discussed in this section. There is also some discussion of most of these line systems in [34].

We start with the Leech lattice $\Lambda$ in 24-dimensional real space. This lattice is the unique 24-dimensional even unimodular lattice, see [18]. Its automorphism group is the group $2 \cdot \mathrm{Co}_{1}$. We scale the lattice in such a way that the vectors of type 2 whose stabilizer in $2 \cdot \mathrm{Co}_{1}$ is isomorphic to $\mathrm{Co}_{2}$ have norm 4 . All the line systems of Theorem 1.1 are related to the lattice $\Lambda$.

Throughout this section we will use the Atlas [17] notation for elements in the groups mentioned below.

\subsection{The 2300 lines in $\mathbb{R}^{23}$ related to $2 \times \mathrm{Co}_{2}$.}

Fix a norm 4 type 2 vector $v$ of the Leech lattice $\Lambda$, and consider all the 4600 type 2 vectors of $\Lambda$ that have inner product -2 with $v$. Projection of these 4600 vectors onto the 23-dimensional space $v^{\perp}$ perpendicular to $v$ yields a set of 4600 vectors that are on 2300 lines. Two of these lines make an angle $\alpha$ with $\cos \alpha= \pm 1 / 3$ or 0 . The setwise stabilizer of $\{v,-v\}$ in $\operatorname{Aut}(\Lambda)$ is isomorphic to $2 \times \mathrm{Co}_{2}$ and acts on the 4600 vectors.

\subsection{The 1408 lines in $\mathbb{R}^{22}$ related to $2 \times \operatorname{PSU}_{6}(2)$.}

In the system of 2300 lines obtained above, the 1408 lines perpendicular to a fixed line form also a tetrahedrally closed line system. The local near hexagon of this line system is isomorphic to the Aschbacher near hexagon related to the group ${ }^{+} \Omega_{6}^{-}(3)$, see $[1,8]$. (Here we follow the notation of [21] for the orthogonal groups.) The group $\mathrm{PSU}_{6}(2)$ is contained in the automorphism group of this set of lines. This line system is not in the conclusion of Theorem 1.1, as it is not regular. Indeed, the 


\section{EXTENDED NEAR HEXAGONS AND LINE SYSTEMS}

local near hexagon $\Gamma_{p}$ is not regular as is shown in [8]. However, we have included it here to make the description of the example of 40 lines in $\mathbb{R}^{10}$ related to $2 \times \operatorname{PSU}_{4}(2)$ easier.

3.3 The 256 lines in $\mathbb{R}^{16}$ related to the group $2^{1+8}: \operatorname{Sp}_{6}(2)$.

An involution of type $2 \mathrm{~A}$ in the group $\mathrm{Co}_{2}$ fixes 256 of the 2300 lines that are all contained in the -1-eigenspace of this involution of dimension 16. The centralizer of such an element is a group $2^{1+8}: \operatorname{Sp}_{6}(2)$. The line system is tetrahedrally closed, and is locally the near hexagon of the $\operatorname{PSp}_{6}(2)$ dual polar space. Inside Aut $(\Lambda)$, the centralizer of an element of type $2 A$ is a group isomorphic to $2^{1+8}: \mathrm{O}_{8}^{+}(2)$. The embedding of $\mathrm{Sp}_{6}(2)$ into $\mathrm{O}_{8}^{+}(2)$ is the spin representation of $\operatorname{Sp}_{6}(2)$; the $\operatorname{PSp}_{6}(2)$ near hexagon is embedded into the $\mathrm{PO}_{8}^{+}(2)$ polar space. This will be used later on.

\subsection{The 120 lines in $\mathbb{R}^{15}$ related to $2 \times \operatorname{PSp}_{6}(2)$.}

If one fixes a line of the 256 lines of the above example, then the 120 lines perpendicular to this fixed line form a tetrahedrally closed system of 120 lines in $\mathbb{R}^{15}$, which is locally a generalized hexagon of order $(2,2)$. The group $2 \times \mathrm{PSp}_{6}(2)$ acts transitively on these lines with line stabilizer $2 \times \mathrm{G}_{2}(2)$.

3.5 The 128 lines in $\mathbb{R}^{16}$ related to $2^{1+7}: \mathrm{G}_{2}(2)$.

Let $\sigma$ be an element of order 4 in the normal 2-group of $2^{1+8}: \operatorname{Sp}_{6}(2)$. Then the centralizer of this element induces a transitive group $2^{7}: \mathrm{G}_{2}(2)$ on the 128 lines fixed by $\sigma$. These 128 lines form a closed line system in the 1-eigenspace of dimension 16 of $\sigma$, which is locally a generalized hexagon of order $(2,2)$ related to $\mathrm{G}_{2}(2)$. Notice that the 128 lines are not contained in the set of 256 lines on which $2^{1+8}: \mathrm{Sp}_{6}(2)$ acts as in example 3.3

\subsection{The 40 lines in $\mathbb{R}^{10}$ related to $2 \times \mathrm{PSU}_{4}(2)$.}

In the group $\mathrm{PSU}_{6}(2)$ an element of type $3 A$ is centralized by a group isomorphic to $\mathrm{PSU}_{4}(2)$. Such an element of type $3 A$ has an eigenspace of dimension 10 in the 22-dimensional representation of $2 \times \mathrm{PSU}_{6}(2)$ on the 1408 lines in the second example of this section. There are 40 lines inside this eigenspace that form a closed line system which is locally the near hexagon of Hamming type on 27 points.

3.7 The systems $C_{10}, C_{16}$, and $C_{28}$ related to $2 \times \operatorname{PSp}_{4}(2), 2^{1+4}: \operatorname{Sp}_{4}(2)$ and $2 \times$ $\mathrm{PSp}_{6}(2)$, respectively. 
The maximal sets of respectively 10, 16 or 28 non perpendicular lines in the examples $3.6,3.3$ or 3.1 yield tetrahedrally closed systems $C_{10}, C_{16}$ and $C_{28}$ in $\mathbb{R}^{5}, \mathbb{R}^{6}$ and $\mathbb{R}^{7}$ respectively. These systems are locally a generalized quadrangle of order $(2, t)$, where $t$ is 1,2 or 4 , respectively.

All the above systems are contained in the system of 2300 lines associated to the second Conway group $\mathrm{Co}_{2}$. The following and last example, however, although closely related to the Leech lattice and the group $\mathrm{Co}_{2}$, is not contained in that system.

\subsection{The 2048 lines in $\mathbb{R}^{24}$ related to $2^{1+11}: \mathrm{M}_{24}$.}

Consider a orthonormal basis $\mathcal{B}$ for $\mathbb{R}^{24}$ with respect to the standard inner product. The set of vectors of the form $\Sigma_{b \in \mathcal{B}} \pm b$ form a 24-dimensional vector space over the field $\{ \pm 1\}$. Inside this vector space we can consider the extended binary Golay code $\mathcal{C}$ as a 12-dimensional subspace. The 4096 vectors inside $\mathcal{C}$ span 2048 lines in $\mathbb{R}^{24}$. The inner product of 2 codewords (as vectors in $\mathbb{R}^{24}$ ) equals \pm 24 (if they are the same or opposite), \pm 8 or 0 . So two distinct lines make an angle $\alpha$ with $\cos \alpha=0$ or $\pm 1 / 3$. The system is tetrahedrally closed. The local near hexagon at each vector is isomorphic to the near hexagon on 759 points related to the group $\mathrm{M}_{24}$. The stabilizer of this line system inside the orthogonal group on $\mathbb{R}^{24}$ with its standard inner product, is isomorphic to the group $2^{1+11}: \mathrm{M}_{24}$.

\section{The structure and combinatorics of extended near hexagons}

In this section we start the investigation of extended near hexagons as in the hypothesis of Theorems 1.2 and 1.3.

Let $\Gamma=(\mathcal{P}, \mathcal{C})$ be an extended near hexagon in which all circles contain at least 4 points. By $\mathcal{G}$ we denote the point graph of $\Gamma$, whose edge set will be denoted by $\mathcal{E}$. We assume that the graph $\mathcal{G}$ is locally the distance 1-or-2 graph of the local near hexagon. So, if $p$ is a point of $\Gamma$, then two points $q$ and $r$ in $\mathcal{P}_{p}$ are adjacent if and only if they are at distance at most 2 in the near hexagon $\Gamma_{p}$. Moreover, for all points $p$ of $\Gamma$ we assume the near hexagon $\Gamma_{p}$ to be regular.

Lemma 4.1 There are parameters $\left(s, t_{2}, t\right)$ such that for all $p \in \mathcal{P}$, the residue $\Gamma_{p}$ is a regular near hexagon with parameters $\left(s, t_{2}, t\right)$.

Proof. Fix a point $p$ and suppose that the near polygon $\Gamma_{p}$ has parameters $\left(s, t_{2}, t\right)$. Let $q$ be a point cocircular with $p$. Suppose that the near hexagon $\Gamma_{q}$ has parameters 
$\left(s^{\prime}, t_{2}^{\prime}, t^{\prime}\right)$. As there are $t+1$ lines on $q$ in $\Gamma_{p}$, there are $t+1$ circles containing both $p$ and $q$. So the point $p$ of $\Gamma_{q}$ is on $t+1=t^{\prime}+1$ lines in $\Gamma_{q}$ and $t=t^{\prime}$. As all circles on $p$ contain $s+2$ points, the lines of $\Gamma_{q}$ on $p$ contain $s+1$ points. Hence $s^{\prime}=s$.

Finally we will prove that $t_{2}^{\prime}=t_{2}$. Consider the set $\left(p^{\perp} \cap q^{\perp}\right)-\{p\}$. This is the set of points at distance at most 2 from $q$ in $\Gamma_{p}$, it has cardinality $1+s(t+1)+s(t+$ 1) $s t /\left(t_{2}+1\right)$. Similarly, $\left(q^{\perp} \cap p^{\perp}\right)-\{q\}$, which is the set of points in $\Gamma_{q}$ at distance at most 2 from $p$ has cardinality $1+s(t+1)+s(t+1) s t /\left(t_{2}^{\prime}+1\right)$. But since these two sets are of the same size, we find $t_{2}^{\prime}=t_{2}$. This implies that $\Gamma_{q}$ has the same parameter set as $\Gamma_{p}$. By connectedness of the point graph of $\Gamma$ we have proved the lemma.

From now on we fix integers $s, t$ and $t_{2}$ such that for all points $p$ of $\Gamma$ the local near hexagon $\Gamma_{p}$ has order $\left(s, t_{2}, t\right)$.

Lemma 4.2 Let $C$ be a circle and $p$ a point of $\Gamma$. Then $C \subseteq p^{\perp}$ or $\left|p^{\perp} \cap C\right|=0$ or 2 .

Proof. Suppose $p$ is a point and $C$ a circle meeting $p^{\perp}$. Fix a point $q \in C \cap p^{\perp}$. Inside $\Gamma_{q}$ the point $p$ is either at distance 3 from all but one of the points of $C-\{q\}$, in which case $p^{\perp} \cap C$ consists of 2 points, or $p$ is at distance at most 2 from every point of $C-\{q\}$, and $C \subseteq p^{\perp}$.

Corollary 4.3 Suppose $p$ and $q$ are points of $\Gamma$ at mutual distance 2 . If $s=2$, then $p^{\perp}-q^{\perp}$, i.e., the complement of $p^{\perp} \cap q^{\perp}$ in $p^{\perp}$, is a geometric hyperplane of $\Gamma_{p}$.

Proof. Suppose $s$ to be equal to 2 . Let $C$ be a circle on $p$, then, by 4.2 , there are 0 or 2 points of $C$ in $q^{\perp}$. So, $C-\{p\}$ is contained in $p^{\perp}-q^{\perp}$ or meets it in just one point. Hence $p^{\perp}-q^{\perp}$ is a geometric hyperplane of $\Gamma_{p}$.

Lemma 4.4 Two circles meet in 0,1 or 2 points.

Proof. Suppose $C$ and $D$ are two distinct circles meeting in at least 3 points. Without loss of generality we may assume that there is a point $q \in D-C$. Fix a point $p \in C \cap D$. Then inside $\Gamma_{p}$ the two circles $C$ and $D$ define two lines with at least two points in common, which is well known to be impossible in a near hexagon.

Lemma 4.5 The parameter s and the size of a circle are both even. 


\section{EXTENDED NEAR HEXAGONS AND LINE SYSTEMS}

Proof. Let $C$ be a circle and $q$ a point outside of $C$ with $C \subseteq q^{\perp}$. (Such a point can be found in $\Gamma_{r}$, where $r$ is a point of $C$.)

If $p$ is a point of $C$, then we see inside $\Gamma_{p}$ that $q$ is collinear to a unique point of $C-\{p\}$. Thus there is a unique circle on $q$ and $p$ meeting $C$ in two points. But that implies that the circles on $q$, meeting $C$ in two points, partition $C$ into pairs. In particular, $|C|=s+2$ is even.

Lemma 4.6 Let $X$ be a set of points in a near hexagon such that the distance between any two points of $X$ is at most 2. If a line of the near hexagon is properly contained in $X$, then $X$ is either contained in a quad or there is a unique point $p \in X$ such that all points of $X$ are collinear with $p$.

Proof. Suppose $X$ is a set as in the hypothesis of the lemma containing some line $l$. We assume that $X \neq l$, so that there is a point $x$ in $X-l$. Since $x$ is at distance at most 2 from any point on $l$, it is at distance 1 from $l$. Let $p$ be the unique point on $l$ collinear with $x$.

So, either every point of $X$ is collinear to $p$ or there is a point in $X$ not collinear to $p$. We assume that we are in the latter case and show that $X$ is contained in some quad. Let $y$ be a point in $X$ not collinear to $p$. Then $y$ is at distance $\leq 2$ from both $p$ and $x$, and thus collinear with some point $z$ on the line through $p$ and $x$. The point $y$ is also at distance one from the line $l$. Hence there is a point $u \in l$ collinear with $y$. Thus $x$ and $l$ are contained in some quad $Q$. As $x$ and $l$ are in at most one quad, $Q$ is the unique quad containing $x$ and $l$. This quad also contains $y$ and $z$.

The above implies that every point of $X$ not collinear to $p$ is contained in the quad $Q$.

Now suppose $v \in X$ is collinear to $p$. As $y$ is at distance at most 2 from both $p$ and $v$, it is collinear to a point on the line through $p$ and $v$. Since $Q$ is geodesically closed, this line and therefore also $v$ is contained in $Q$.

In a regular near hexagon with parameters $\left(s, t_{2}, t\right)$ where $s \geq 2$, Yanuska's Lemma shows that the existence of quads is equivalent with $t_{2}>0$. This will be exploited below.

Lemma 4.7 If $t_{2} \geq 1$, and $p$ is a point of $\Gamma$, then for all quads $Q$ of $\Gamma_{p}$, the set $\{p\} \cup Q$ together with the circles contained in it, is a one point extension of a generalized quadrangle.

Proof. Fix a point $p$ of $\Gamma$, and suppose $Q$ is a quad of the near hexagon $\Gamma_{p}$. Then $E:=\{p\} \cup Q$ is a clique in the point graph $\mathcal{G}$ of $\Gamma$. Suppose $q$ is a point in $E$ 
different from $p$. Then consider the point set $E-\{q\}$ in $\Gamma_{q}$. This is a subset of the near hexagon $\Gamma_{q}$ satisfying the hypothesis of the previous lemma. The point $p$ is on $t_{2}+1$ lines in this set. Thus by the above lemma either all points are collinear with $p$, or $E-\{q\}$ is contained in a quad. Since there are $(1+s)\left(1+s t_{2}\right)$ points in $E-\{q\}$ of which there are only $s\left(t_{2}+1\right)$ points collinear with $p$, this set $E-\{q\}$ is contained in a quad; it even consists of all points of a quad of $\Gamma_{q}$. This proves the lemma.

Corollary 4.8 If $t_{2} \geq 1$, then $s+2$ divides $2 t_{2}\left(t_{2}+1\right)\left(2 t_{2}-1\right)$.

Proof. This follows from the above lemma and [14, Theorem 5.1].

The extended generalized quadrangles, as considered in the above lemma, will be called extended quads of $\Gamma$. By $\mathcal{E} \mathcal{Q}$ we denote the set of all extended quads of $\Gamma$. A consequence of the above lemma is that the extension of a near hexagon with quads (i.e., a near hexagon with $t_{2}>0$ ) under consideration is a Buekenhout geometry with diagram

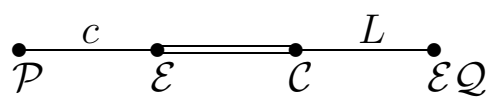

Lemma 4.9 If $t_{2}>0$, then $2+s\left(t_{2}+1\right) \mid\left(s^{2}+2 s+2\right)(1+s)^{2} s\left(t-t_{2}\right)$ and $2+s t_{2} \mid s^{3}\left[\frac{t(t+1)-\left(t-t_{2}\right)\left(t_{2}+1\right)^{2}}{t_{2}\left(t_{2}+1\right)}-1\right]$.

Proof. Suppose $t_{2}>0$. Then, as we saw above, $\Gamma$ contains extended quads. Let $E Q$ be such an extended quad and fix a point $p \in E Q$. Now consider a point $q$ adjacent to $p$ but not in $E Q$. Denote by $Q_{p}$ the quad of $\Gamma_{p}$ contained in $E Q$. Within the near hexagon $\Gamma_{p}$ we see that $q$ is either collinear to a point of $Q$ or at distance $\geq 2$ from all points of $Q$ and the points of $Q$ at distance 2 from $q$ form an ovoid of $Q$. In the first case $q$ is adjacent to $1+1+s\left(t_{2}+1\right)$ points of $E Q$, in the second case to $1+1+s t_{2}$ points.

Suppose $q^{\perp} \cap E Q$ contains $1+1+s\left(t_{2}+1\right)$ points. Let $p^{\prime}$ be a point $E Q$ different from $p$ but adjacent to $q$. Denote by $Q_{p^{\prime}}$ the quad of $\Gamma_{p^{\prime}}$ contained in $E Q$. Then, inside $\Gamma_{p^{\prime}}$ the number of points of $Q^{\prime}$ at distance $\leq 2$ from $q$ equals $1+s\left(t_{2}+1\right)$. So, inside $\Gamma_{p^{\prime}}$ we see that $q$ is at distance 1 from the quad $E Q_{p^{\prime}}$.

Counting pairs of adjacent points $x, y$ with $x \in E Q$ and $y \notin E Q$, but $y$ at distance 1 from the quad $E Q_{x}$ inside $\Gamma_{x}$ yields $\left[1+(1+s)\left(1+s t_{2}\right)\right] \cdot(1+s)(1+$ 
$\left.s t_{2}\right) s\left(t-t_{2}\right)=\alpha \cdot\left(2+s\left(1+t_{2}\right)\right)$, where $\alpha$ is the number of points $y$ outside $E Q$ but with $y^{\perp} \cap E Q$ of cardinality $2+s\left(t_{2}+1\right)$. Hence $2+s\left(t_{2}+1\right) \mid\left[1+(1+s)\left(1+s t_{2}\right)\right] \cdot(1+$ $s)\left(1+s t_{2}\right) s\left(t-t_{2}\right)$ from which we deduce that $2+s\left(t_{2}+1\right) \mid\left[1+(1+s)^{2}\right] \cdot(1+s)^{2} s\left(t-t_{2}\right)$.

By counting pairs of adjacent points $x, y$ with $x \in E Q$ and $y \notin E Q$, but $y$ at distance 2 from the quad $E Q_{x}$ inside $\Gamma_{x}$ we obtain the second divisibility condition.

Let $p$ and $q$ be two adjacent points of $\Gamma$ and denote by $X_{p, q}$ the union of all circles on $p$ and $q$. This is a clique in the point graph of $\Gamma$ containing $1+1+s(t+1)$ points.

Lemma 4.10 Let $p$ and $q$ be adjacent points in $\Gamma$ and suppose $x$ is a point of $X_{p, q}$. Then there is a point $y$ in $X_{p, q}$ different from $x$ with $X_{x, y}=X_{p, q}$.

Proof. If $x$ equals $p$ or $q$, then the result is obviously true. Thus assume $x$ to be different from both $p$ and $q$. The set $X_{p, q}-\{x\}$, as subset of $\Gamma_{x}$, satisfies the conditions of Lemma 4.6. Moreover, as $X_{p, q}$ is a maximal clique, $X_{p, q}-\{x\}$ is either a quad in $\Gamma_{x}$ or there is a point $y$ of $\Gamma_{x}$ such that $X_{p, q}-\{x\}$ consists of points of $\Gamma_{x}$ collinear to $y$. If we are in the first case, then Lemma 4.7 implies that $X-\{p\}$ is also a quad, which, however, is not the case. So we are in the second case and $X_{p, q}$ is contained in $X_{x, y}$ for some point $y$ of $\Gamma_{x}$. But as $X_{p, q}$ and $X_{x, y}$ both have size $2+s(t+1)$, we obtain $X_{x, y}=X_{p, q} \cdot \square$

Corollary $4.11 s+2$ divides $2 t(t+1)$.

Proof. Let $p$ and $q$ be cocircular points and consider $X=X_{p, q}$. Each point of $X$ is on $t+1$ circles contained in $X$. As each circle contains $s+2$ points, there are $(2+s+s t)(1+t) /(s+2)$ circles contained in $X$. Thus $s+2$ divides $(2+s+s t)(1+t)$ and hence also $2 t(t+1)$.

Corollary 4.12 If $s=2$, then the symmetric difference of two circles meeting in two points is again a circle.

Proof. Suppose $C$ and $D$ are two circles meeting in two points $p$ and $q$. Consider the two points $p^{\prime}$ and $q^{\prime}$ on $C$ distinct from $p$ and $q$. By Lemma 4.10 we find that $X_{p, q}=X_{p^{\prime}, q^{\prime}}$. In particular, there is a circle $E$ on $p^{\prime}$ and $q^{\prime}$ different from $C$ and meeting $D$ non trivially. Since two circles meet in 0 or 2 points, $E$ meets $D$ in the two points different from $p$ and $q$. In particular, $E$ is the symmetric difference of the two circles $C$ and $D$. 


\section{EXTENDED NEAR HEXAGONS AND LINE SYSTEMS}

Lemma 4.13 Let $p$ and $q$ be two points of $\Gamma$ at distance 2 . If $r$ is a point in $p^{\perp}-q^{\perp}$, then there are $0,\left(2+s+s t_{2}\right) / 2$ or $(2+t) / 2$ circles through $p$ and $r$ meeting $q^{\perp}$ non trivially. Moreover, the last possibility does occur, only if $s>2$.

Proof. Consider the set $X_{p, r}$ consisting of all points cocircular with $p$ and $r$. Suppose there is a point $x \in X_{p, r} \cap q^{\perp}$ different from $p$ and $r$. By Lemma 4.10 there is a unique point $y$ in $X_{p, r}$ different from $x$ with $X_{x, y}$ equal to $X_{p, r}$. If $q$ and $y$ are at distance 2 in $\Gamma_{x}$, then $q^{\perp}$ meets $X_{x, y}$ in $2+s\left(t_{2}+1\right)$ points. If $q$ and $y$ are at distance 3 , then $q^{\perp}$ meets $X_{x, y}$ in $1+t+1$ points. Since each circle on $p$ and $r$ has either 0 or 2 points in $q^{\perp}$, there are $\left(2+s+s t_{2}\right) / 2$ or $(t+2) / 2$ circles on $p$ and $q$ meeting $q^{\perp}$ non trivially.

Now suppose $s=2$. Then $C=\{p, r, x, y\}$ is a circle meeting $q^{\perp}$ in two points, i.e., in the points $x$ and $y$. Hence $y$ and $q$ are at distance 2 in $\Gamma_{x}$, and the second part of the lemma follows.

As noticed in the introduction, we say that a near hexagon of order $\left(s, t_{2}, t\right)$ has classical parameters if either $t_{2}>0$ and $t=t_{2}\left(t_{2}+1\right)$, or $t_{2}=0$ and $t \in\left\{1, \sqrt[3]{s}, s, s^{3}\right\}$. Combining various of the above results we obtain:

Theorem 4.14 If $\Gamma$ is an extension of a regular near hexagon with classical parameters $\left(s, t_{2}, t\right), s \geq 2$, then $s=2$.

Proof. Let $\Gamma$ be an extension of a regular near hexagon with parameters $\left(s, t_{2}, t\right)$, $s \geq 2$ and $t \geq 1$. Suppose $p$ and $q$ are two cocircular points and set $X=X_{p, q}$. A point $x \in \Gamma_{p}$ but not in $X$ has $t+2$ neighbors in $X$ if it is at distance 3 from $q$. Let $x$ be such a point (there are $s^{3} t\left(t-t_{2}\right) /\left(t_{2}+1\right)$ of them), and suppose $y$ is a neighbor of $x$ in $X$. On the circle $C$ that contains $p, q$ and $y$ the two neighbors of $x$ are just $p$ and $y$. So, if $z$ is the unique point in $X$ different from $y$ with $X=X_{y, z}$, then $x$ and $z$ are not adjacent, and inside $\Gamma_{y}$ the points $x$ and $z$ are at distance 3 . This implies that $x^{\perp}$ meets each circle in $X$ in just 0 or 2 points.

Hence the number of points adjacent to some point of $X$ but not to all the points of a circle in $X$ equals $(2+s+s t) s^{3} t\left(t-t_{2}\right) /\left((t+2)\left(t_{2}+1\right)\right)$. In particular, $t+2$ divides $(2+s+s t) s^{3} t\left(t-t_{2}\right)$ and then also $2(s-2) s^{3}\left(t_{2}+2\right)$.

Suppose $s>2$. First assume that $t_{2}=0$ and $t$ equals $1, \sqrt[3]{s}, s$ or $s^{3}$. Then $\Gamma$ is an extension of a generalized hexagon. Since $s+2$ divides $2 t(t+1)$, we find that $t$ can not be equal to $1, \sqrt[3]{s}$ or $s$, but has to be $s^{3}$. However, this contradicts that $t+2$ divides $4(s-2) s^{3}$.

Next assume that $t_{2}>0$ and $t=t_{2}\left(t_{2}+1\right)$. For each point $p$ of $\Gamma$ the local near hexagon $\Gamma_{p}$ is either of Hamming type and $t_{2}=1$, or is a dual polar space and $t_{2}$ equals $\sqrt[2]{s} \sqrt[3]{s^{2}}, s$, or $s^{2}$. In the latter case $s$ and $t_{2}$ are powers of 2 , see [13]. 
Since $s+2$ divides $2 t_{2}\left(t_{2}+1\right)\left(2 t_{2}-1\right)$, see 4.8 , we find that $t_{2}$ is at least 2 . Moreover, using the above, 4.8 and 4.11 , we find that $s+2$ divides $4\left(t_{2}+1\right)\left(2 t_{2}-1\right)$ and $4\left(t_{2}+1\right)\left(t_{2}^{2}+t_{2}+1\right)$. Since $4 \cdot\left(t_{2}^{2}+t_{2}+1\right)-\left(2 t_{2}+3\right) \cdot\left(2 t_{2}-1\right)=7$, we deduce that $s+2$ divides $28\left(t_{2}+1\right)$.

If $t_{2}=\sqrt{s}$, then $s+2 \mid 28(\sqrt{s}+1)$ implies that $s=4$ and $t_{2}=2$. However, then 4.9 implies that $2+s\left(t_{2}+1\right)=14$ divides $\left[1+(1+s)^{2}\right] \cdot(1+s)^{2} s\left(t-t_{2}\right)=26 \cdot 25 \cdot 4 \cdot 2^{2}$, a contradiction.

If $t_{2}=\sqrt[3]{s^{2}}$, then $s+2 \mid 28\left(\sqrt[3]{s^{2}}+1\right)$ implies that $s=8$ and $t_{2}=4$.

If $t_{2}=s$, then $s+2 \mid 28\left(t_{2}+1\right)$ implies $s+2 \mid 28$, which contradicts $s$ to be a power of 2 .

If $t_{2}=s^{2}$, then $s+2 \mid 28\left(t_{2}+1\right)$ implies $s+2 \mid 140$. Here we can conclude that $\left(s, t_{2}\right)=(8,64)$.

Hence, we are left with the cases that $\left(s, t_{2}\right)$ equals $(8,4)$ or $(8,64)$. However, in both cases $t+2$ does not divide $2(s-2) s^{3}\left(t_{2}+2\right)$. This contradiction proves the theorem.

\section{Extension of near hexagons with three points per line}

In this section we consider extensions of near hexagons as in the hypothesis of Theorem 1.2. Let $\Gamma$ be such an extended near hexagon. Then, conform Lemma 4.1, there exist $t_{2}$ and $t$ such that for each point $p$ of $\Gamma$ the local near hexagon $\Gamma_{p}$ is a regular near hexagon of order $\left(2, t_{2}, t\right)$. By $[7,8]$, we either have that this near hexagon is a generalized quadrangle of order $(2, t)$, with $t=1,2$ or 4 , a generalized hexagon of order $(2, t)$, with $t=1,2,8$, or one of the following near hexagons containing quads: the near hexagon of Hamming type on 27 points, the near hexagons on 135, respectively, 891 points associated to the dual polar spaces related to $\operatorname{PSp}_{6}(2)$ and $\mathrm{PSU}_{6}(2)$, respectively, the $\mathrm{M}_{24}$-near hexagon on 759 points or the near hexagon on 729 points related to $3^{6}: 2 \cdot \mathrm{M}_{12}$.

In general, the approach for proving Theorem 1.2 is the following. Suppose $\Gamma$ is an extension of a near hexagon with 3 points per line. We first consider the point graph $\mathcal{G}$ of $\Gamma$. This graph is locally the distance 1-or-2 graph of the local near hexagon. The second neighborhood of a point $p$, i.e., the subgraph of $\mathcal{G}$ consisting of the vertices at distance 2 from $p$, will be identified with the use of $\mu$-graphs, or rather their complements. The subgraph of $\mathcal{G}$ induced on the second neighborhood of $p$ can be recovered by classifying the various $\mu$-graphs and considering the intersections between these $\mu$-graphs. This approach eventually leads to the determination of the isomorphism type of $\mathcal{G}$. To finish the proof, we have to consider the various ways one 
can fix a collection of 4-cliques of $\mathcal{G}$, such that these 4-cliques induce the structure of an extended near hexagon on $\mathcal{G}$. This program will be carried out for the several possibilities of the local near hexagon $\Gamma_{p}$.

The main tool in the classification of $\mu$-graphs is the following proposition in which we collect some properties of the complement of a $\mu$-graph. For the first two properties, see 4.3 and 4.13 ; the third property follows easily from the second one.

Proposition 5.1 Let $p$ and $q$ be points of $\Gamma$ at mutual distance 2 . Then we have the following:

i. $p^{\perp}-q^{\perp}$ is a geometric hyperplane of $\Gamma_{p}$;

ii. if $r$ is a point of the hyperplane $p^{\perp}-q^{\perp}$ of $\Gamma_{p}$, then either $2+t_{2}$ lines of $\Gamma_{p}$ on $r$ meet $q^{\perp}$ or $r$ is a deep point of $p^{\perp}-q^{\perp}$.

iii. if for all $x$ and $y$ at mutual distance 2 in $\mathcal{G}$ the hyperplane $x^{\perp}-y^{\perp}$ of $\Gamma_{x}$ does not contain deep points, then the diameter of the graph $\mathcal{G}$ equals 2.

\subsection{Extensions of generalized quadrangles}

In this subsection we consider extensions of the generalized quadrangles of order 2. These are one point extensions. Such extensions have been studied by several authors, and a proof of the following result can be found at various places, see for example [14].

Proposition 5.2 Suppose $\Gamma$ is a one point extension of a generalized quadrangle with $s=2$, then it is one of the three unique extensions on 10, 16 or 28 points related to the groups $\mathrm{PSp}_{4}(2), 2^{4}: \operatorname{Sp}_{4}(2)$, respectively, $\mathrm{PSp}_{6}(2)$.

\subsection{Extensions of generalized hexagons}

Now we assume that for all $p$ of $\Gamma$, the local space $\Gamma_{p}$ is a generalized hexagon with 3 points per line. By a result of Haemers and Roos, see [7], we have that $t$ can only take the values 1,2 or 8 . The generalized hexagons with these parameters have been classified, see [16]. There is only one generalized hexagon of order $(2,1)$, its point graph is the flag-graph of the Fano plane. There are two generalized hexagons of order $(2,2)$, denoted by $\mathcal{H}$ and $\mathcal{H}^{*}$. They are dual to each other and are the classical generalized hexagons related to the group $\mathrm{G}_{2}(2)$. We distinguish between $\mathcal{H}$ and $\mathcal{H}^{*}$ in the following way: $\mathcal{H}$ is the $\mathrm{G}_{2}(2)$ generalized hexagon for which the stabilizer in $\mathrm{G}_{2}(2)$ of a point is of the shape $4^{2}: \mathrm{D}_{12}$, while the stabilizer of a point of $\mathcal{H}^{*}$ is of the shape $2^{1+4}: \mathrm{S}_{3}$. Geometrically these two generalized hexagons can be distinguished in the following way. The hexagon $\mathcal{H}$ contains subhexagons of order 


\section{EXTENDED NEAR HEXAGONS AND LINE SYSTEMS}

$(2,1)$, while $\mathcal{H}^{*}$ does not contain such hexagons. Related to the group ${ }^{3} \mathrm{D}_{4}(2)$ there is a generalized hexagon of order $(2,8)$; it is the unique generalized hexagon with parameters $(s, t)=(2,8)$.

Lemma 5.3 For all points $p$ of $\Gamma$, the local generalized hexagon $\Gamma_{p}$ has order $(2,2)$.

Proof. Suppose $\Gamma$ is locally of order $(2, t)$, with $t \in\{1,2,8\}$. Fix a point $p$ of $\Gamma$, and let $q$ be a point at distance 2 from $p$. Then, by Proposition 5.1, the complement of the $\mu$-graph $p^{\perp} \cap q^{\perp}$ is a geometric hyperplane of the generalized hexagon $\Gamma_{p}$. Call this hyperplane $H$. It follows by 5.1 that each point of this hyperplane $H$ is on $t-1$ lines contained in the hyperplane, or all lines on the point are in the hyperplane.

So, if $t=1$, then obviously $H$ can not contain deep points, and we find $H$ to be an ovoid. In particular, counting collinear point pairs $(u, v)$ with $u \in H$ and $v \notin H$ in two ways, we find $4|H|=2(21-|H|)$; so $H$ contains 7 points. This implies that $\mathcal{G}$ is a strongly regular graph with parameters $(k, \lambda, \mu)=(21,12,14)$. However, these parameters do not satisfy the feasibility conditions for a strongly regular graph, see [7].

Suppose $t=8$. Then, again by counting collinear point pairs $(u, v)$ with $u \in H$ and $v \notin H$, we have $9(819-|H|)=4(|H|-|D|)$, where $D$ is the set of deep points in $H$. From this it follows that $|H|=567+4|D| / 13$.

Fix a line $l=\{u, v, w\}$ of the near hexagon meeting $H$ in the point $u$. Suppose that $u$ is collinear to $x$ deep points of $H$. Then $H$ contains 1 point of $l$, it contains $16+14$ points at distance 1 from $l$, and $536+4 x$ points at distance 2 from the line $l$. Hence $H$ contains $567+4 x$ points, and $x=|D| / 13$. (Notice that this implies that $x$ is independent of the choice of the point $u$.) In particular, $|D|=13 x \leq 13 \cdot 14$. If $x>0$, then counting the collinear pairs $(v, w)$ in $H$, with $v$ deep and $u$ not, we obtain that $(|H|-|D|) x \leq|D| \cdot 18$, and hence $|H| \leq|D|+(|D| / x) \cdot 18 \leq 13 \cdot 14+13 \cdot 18<567$, a contradiction. Thus $x=0$ and we can assume that $H$ does not contain deep points. But that implies that $\mathcal{G}$ is a strongly regular graph with $k=819, \lambda=306$ and $\mu=252$. In particular, the number of points equals 2484 .

By 4.10 each point $p$ of $\Gamma$ is in 819 maximal cliques $X_{p, q}$ of size 20. Let $\alpha$ denote the number of such maximal cliques. Counting the number of pairs $\left(p, X_{p, q}\right)$ in two ways yields that $\alpha \cdot 20=2484 \cdot 819$, which is impossible. This contradiction implies that $t \neq 8$, and the lemma is proved. $\square$

In the remainder of this subsection we assume $\Gamma$ to be locally a generalized hexagon of order $(2,2)$.

Lemma 5.4 Let $p$ and $q$ be points of $\Gamma$. If the distance between $p$ and $q$ is 2 , then the complement of $p^{\perp} \cap q^{\perp}$ in $\Gamma_{p}$ contains 27,35 or 31 points. 


\section{EXTENDED NEAR HEXAGONS AND LINE SYSTEMS}

Proof. Consider two points $p$ and $q$ that are at distance 2 in the graph $\mathcal{G}$. The complement of $p^{\perp} \cap q^{\perp}$ in $\mathcal{P}_{p}$ is a geometric hyperplane of $\Gamma_{p}$, and will be denoted by $H$. It follows from Proposition 5.1 that each point in $H$ is either deep, or is on just one line inside the hyperplane. Let $D$ be the set of deep points in $H$. Then by counting the pairs of collinear points $(u, v)$ with $u \in H$ and $v \notin H$ we find $(|H|-|D|) 4=(63-|H|) 3$. Fix a point $u \in H-D$, that is collinear with $x$ deep points, $x=0,1$ or 2 . Then there are 2 points at distance 1 from $u$ in $H$, there are $4 x+8$ points in $H$ at distance 2 from $u$, and, finally, there are 16 points at distance 3 from $u$ in $H$. Hence $|H|=27+4 x=27+4|D| / 7$. So every point $u \in H-D$ is adjacent to $|D| / 7$ deep points, and $|D|=0,7$ or 14 . In particular, $|H|=27,31$ or 35. ㅁ

Lemma 5.5 For all points $p$ and $q$ of $\Gamma$, the generalized hexagons $\Gamma_{p}$ and $\Gamma_{q}$ are isomorphic.

Proof. Suppose that there is a point $p$ in $\Gamma$ with $\Gamma_{p}$ isomorphic to $\mathcal{H}^{*}$.

Fix a point $q$ at distance 2 from $p$. The previous lemma implies that the complement $H$ of $p^{\perp} \cap q^{\perp}$ is a geometric hyperplane of $\Gamma_{p}$ containing 27,35 or 31 points. By $[25], \mathcal{H}^{*}$ does not contain hyperplanes on 27 or 35 points. So $H$ contains 31 points. Moreover, the results of [25] imply that $H$ consists of all the points in $\Gamma_{p}$ at distance at most 2 from some fixed point of $\Gamma_{p}$.

The distance 1-or-2 graphs induced on the set of points at distance 3 in the two generalized hexagons $\mathcal{H}$ and $\mathcal{H}^{*}$ are non isomorphic. So, as $p^{\perp} \cap q^{\perp}$ is isomorphic to such a graph in $\Gamma_{p} \simeq \mathcal{H}^{*}$, we can conclude that $\Gamma_{q}$ is also isomorphic to $\mathcal{H}^{*}$.

Let $r$ be a point in $p^{\perp}$. Fix a point $r^{\prime}$ in $\Gamma_{p}$ collinear to $r$. Now let $q^{\prime}$ be a point in $\Gamma_{r^{\prime}}$ at distance 3 from $p$ and $r$. Then inside $\Gamma$ the distance between $q^{\prime}$ and $p$ and $r$ is 2 .

The above implies that $\Gamma_{r} \simeq \Gamma_{q^{\prime}} \simeq \Gamma_{p} \simeq \mathcal{H}^{*}$. The connectedness of $\Gamma$ implies now that the residue at each point of $\Gamma$ is isomorphic to $\mathcal{H}^{*}$.

Lemma 5.6 The generalized hexagon $\mathcal{H}^{*}$ can uniquely be recovered from its distance 1-or-2 graph.

Proof. Let $H^{*}$ be the distance 1-or-2 graph of $\mathcal{H}^{*}$. Let $x$ and $y$ be two vertices of $H^{*}$. If $x$ and $y$ are collinear in $\mathcal{H}^{*}$, then $\left(x^{\perp} \cap y^{\perp}\right)^{\perp}$ consists of the 3 vertices $x, y$ and $z$ forming a line of $\mathcal{H}^{*}$. If $x$ and $y$ are non collinear, then $\left(x^{\perp} \cap y^{\perp}\right)^{\perp}$ consists of just $x$ and $y$, see $[35,3.5 .7]$. So the lines of $\mathcal{H}^{*}$ can uniquely be recovered from the graph $H^{*}$. This proves the lemma. 
Lemma 5.7 Let $p$ be a point of $\Gamma$. Then $\Gamma_{p}$ is isomorphic to $\mathcal{H}$.

Proof. Let $p$ be a point of $\Gamma$ and $q$ a point at distance 2 from $p$. Suppose $\Gamma_{p}$ is isomorphic to $\mathcal{H}^{*}$. Then, by the above lemma, $\Gamma_{q}$ is isomorphic to $\mathcal{H}^{*}$.

The results of [25] and Lemma 5.4 imply that the $\mu$-graph $p^{\perp} \cap q^{\perp}$ consists of all the points of $\Gamma_{p}$ at distance 3 (inside $\Gamma_{p}$ ) from some point $r \in \Gamma_{p}$. Thus there are $63 \cdot 32 / 32=63$ points at distance 2 from $p$ (and similarly from $q$ in $\mathcal{G}$ ). We claim that the point $r$ is at distance 3 from $q$ in $\mathcal{G}$. Suppose not, then $q$ and $r$ are at distance 2 and $r^{\perp} \cap q^{\perp}$ consists of the 32 points of $\Gamma_{r}$ opposite to $p$. As $r^{\perp} \cap q^{\perp} \cap p^{\perp}$ is empty, we have found $64=32+32$ points in $\Gamma_{q}$. A contradiction. Hence, $r$ is indeed at distance 3 from $q$.

Next we show that $r$ is the unique point of $\mathcal{G}$ at distance 3 from $q$. Suppose $u$ is a common neighbor of $p$ and $r$. Then $u$ is adjacent to some point in $p^{\perp} \cap q^{\perp}$. Thus $u$ is at distance 2 from $q$. So, we can apply the above to $u$ instead of $p$. The connectedness of $\Gamma_{r}$ implies that all the points of $\Gamma_{r}$ are at distance 2 from $q$. In particular, $\Gamma_{r}$ consists of all the 63 points of $\mathcal{G}$ that are at distance 2 from $q$. Moreover, $r$ is the unique neighbor of each of these 63 points which is at distance 3 from $q$. This implies that $\Gamma$ contains $1+63+63+1$ points; its distance distribution diagram reads as follows:

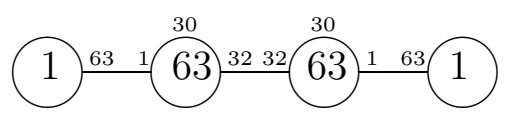

In particular, for each point $x \in \Gamma$ there is a unique point $\bar{x}$ at distance 3 from $x$ in $\mathcal{G}$.

Suppose $x \in \Gamma_{p}$. Then $\bar{x}$ is at distance 2 from $p$. Moreover, $\bar{x}^{\perp} \cap p^{\perp}$ consists of all the points of $\Gamma_{p}$ which are in $\Gamma_{p}$ opposite to $x$.

Suppose that $x$ and $y$ are two points in $\Gamma_{p}$. Then consider the points $\bar{x}$ and $\bar{y}$. If $x$ and $y$ are opposite in $\Gamma_{p}$, then $x$ and $\bar{y}$ are adjacent as well as $y$ and $\bar{x}$. Since $x$ and $\bar{x}$ are at distance 3 , we find that also $\bar{x}$ and $\bar{y}$ are not adjacent. But that implies that $\bar{x}$ and $\bar{y}$ are adjacent if and only if $x$ and $y$ are adjacent. Thus we can describe all adjacencies in $\mathcal{G}$ in terms of $\Gamma_{p}$. The adjacencies within $p^{\perp}$ are clear. The point $\bar{p}$ is adjacent to all points $\bar{x}$, with $x \in \Gamma_{p}$; the point $\bar{x}, x \in \Gamma_{p}$, is adjacent to $\bar{p}$, and all points $y$ of $\Gamma_{p}$ at distance 3 from $x$ in $\Gamma_{p}$, and finally $\bar{x}$ and $\bar{y}$, where $x, y$ are points of $\Gamma_{p}$, are adjacent if and only if $x$ and $y$ are adjacent.

This description shows that the automorphism group $G=\operatorname{Aut}(\mathcal{G})$ of $\mathcal{G}$ contains a subgroup stabilizing $p$ and $\bar{p}$ and inducing $\mathrm{G}_{2}(2)$ on $\Gamma_{p}$ and $\Gamma_{\bar{p}}$. As the point $p$ is arbitrary, we see that the group $G$ is transitive and of permutation rank 4 on $\mathcal{G}$. 
Now consider the stabilizer $G_{p}$ of $p$ inside $G$. As the generalized hexagon $\mathcal{H}^{*}$ can be recovered from its distance 1-or-2 graph, see Lemma 5.6 we find that $G_{p} \simeq \mathrm{G}_{2}(2)$. Let $g \in G_{p}$ be an element of type $3 A$ in the notation of the Atlas [17]. Then $g$ fixes 9 points of the local hexagon $\Gamma_{p}$, and thus 20 points of $\Gamma$. Moreover, every element of order 3 in $G$ fixing exactly 20 points of $\Gamma$ has to be conjugate to $g$. Hence, as $G_{p}$ contains 28 conjugates of $\langle g\rangle$, the number of conjugates of $\langle g\rangle$ in $G$ equals $128 \cdot 28 / 20$, which is impossible. This final contradiction shows that for all $p$ in $\Gamma$, the local generalized hexagon $\Gamma_{p}$ is isomorphic to $\mathcal{H}$.

So, for the remainder of this subsection we may and do assume that for all points $p$ of $\Gamma$, the local generalized hexagon $\Gamma_{p}$ is isomorphic to $\mathcal{H}$. This implies that the graph $\mathcal{G}$ is locally isomorphic to the distance 1-or-2 graph of $\mathcal{H}$. This graph is isomorphic to the point graph of the symplectic polar space related to $\operatorname{PSp}_{6}(2)$. From this observation we deduce:

Proposition 5.8 The graph $\mathcal{G}$ is isomorphic to the collinearity graph of an affine polar space obtained from the $\mathrm{PSp}_{8}(2)$ polar space by removing a hyperplane.

Proof. This follows by the results of $[15,26]$ or $[20]$.

Let $\mathcal{S}$ be the $\mathrm{PSp}_{8}(2)$ polar space. Up to isomorphism, there are three types of hyperplanes in the $\mathrm{Sp}_{8}(2)$ polar space, the degenerate ones and the $\mathrm{PO}_{8}^{+}(2)$ and $\mathrm{PO}_{8}^{-}(2)$ hyperplanes, see [15]. Thus $\mathcal{G}$ is isomorphic to the collinearity graph of an affine polar space $\mathcal{A S}$ obtained from the $\mathrm{Sp}_{8}(2)$ polar space $\mathcal{S}$ by removing a degenerate hyperplane, a $\mathrm{PO}_{8}^{+}(2)$ hyperplane, or a $\mathrm{PO}_{8}^{-}(2)$ hyperplane.

By the above proposition, it remains to check which of the three graphs does contain a set of distinguished 4-cliques making it into the point graph of an extended hexagon. Let $\mathcal{A}$ be the (unique) set of affine planes that make $\mathcal{G}$ into the affine polar space $\mathcal{A S}$. Then, as has been shown in $[15,20]$ and [19], there is an equivalence relation, called parallelism on the set of $\mathcal{A}$ of affine planes, whose equivalence classes form (part of) the line set of the polar space at infinity $\mathcal{A S} \mathcal{S}_{\infty}$ of $\mathcal{A S}$. The set $\mathcal{C}$ of circles of $\Gamma$ is a subset of $\mathcal{A}$. In the lemma below we will show that it consists of a union of parallel classes of $\mathcal{A}$.

As a consequence of this observation we see that also for $\Gamma$ we can define a space at infinity, called $\Gamma_{\infty}$, embedded into the polar space at infinity $\mathcal{A S}_{\infty}$ of $\mathcal{A S}$. The space $\Gamma_{\infty}$ consists of all the points of $\mathcal{A S}_{\infty}$, except for the radical of this polar space, and all the lines at infinity corresponding to those parallel classes of affine planes that contain circles of $\mathcal{C}$.

Lemma 5.9 If $\pi$ is a circle in $\mathcal{C}$, then the whole parallel class of $\pi$ in $\mathcal{A}$ is in $\mathcal{C}$. 
Proof. Let $\pi_{0}$ be a plane of $\mathcal{C}$. Let $p$ be a point of $\pi_{0}$ and $q$ a point in $\pi_{0}^{\perp} \backslash \pi_{0}$. By $m$ we denote the line through $p$ and $q$. In the hexagon $\Gamma_{p}$ we see that $m$ is at distance 1 from some line $n$ on $p$ inside $\pi_{0}$. Thus there is a plane $\pi_{1} \in \mathcal{C}$ on $m$ meeting $\pi_{0}$ in $n$. Let $\pi_{2}$ be the affine plane of $\mathcal{A S}$ on $q$ which is parallel to $\pi_{0}$. Fix a line $g$ on $q$ in $\pi_{2}$, but outside $\pi_{1}$. In $\Gamma_{q}$ we see that $g$ is at distance 1 from some element of $\pi_{1}$, (indeed, $\pi_{0}$ and $g$ are in a singular 3-space of $\mathcal{S}$ ) so there is a unique plane $\pi_{3} \in \mathcal{C}$ on $g$ meeting $\pi_{1}$ in a line, $k$ say. Suppose $k$ meets $n$ at the point $r$ of $\Gamma$. Then inside $\Gamma_{r}$ we encounter the three planes $\pi_{0}, \pi_{1}$ and $\pi_{3}$. Since the planes are inside an affine 3-space, we find that $\pi_{0}$ and $\pi_{3}$ intersect in a line on $r$ distinct from $k$ and $n$. In particular, the three planes form a triangle in $\Gamma_{r}$. This contradicts $\operatorname{Res}(r)$ to be a generalized hexagon. Thus $k$ and $n$ meet at infinity. In particular, $\pi_{2}=\pi_{3} \in \mathcal{C}$. We can conclude that the unique plane on $q$ which is parallel with $\pi_{0}$ is also in $\mathcal{C}$.

Let $\pi_{0} \in \mathcal{C}$ and $\pi_{1} \in \mathcal{A}$ be two parallel planes in $\mathcal{A S}$. Let $l_{\infty}$ be the line at infinity in $\mathcal{A S} \mathcal{S}_{\infty}$ of $\pi_{0}$ and $\pi_{1}$. If $\pi_{1}$ contains a point $r$ with $r \in \pi_{0}^{\perp}$ then, by the above, $\pi_{1}$ is also in $\mathcal{C}$. By connectedness of the affine polar space on the singular planes of $S$ meeting $\mathcal{A S}_{\infty}$ just in $l_{\infty}$ and transitivity of being parallel we find that in any case $\pi_{1}$ is in $\mathcal{C}$.

We consider the three possibilities for $\mathcal{G}$ separately. First assume $\mathcal{G}$ to be isomorphic to the graph on 136 points obtained by removing a $\mathrm{PO}_{8}^{-}(2)$ hyperplane from the polar space $\mathcal{S}$. Then each parallel class of circles contains 10 circles. As there are $136 \cdot 63 / 4$ circles in $\Gamma$, we find $34 \cdot 63 / 10$ parallel classes of circles of $\Gamma$, which is impossible.

Now assume that $\mathcal{G}$ is isomorphic to the graph on 120 points obtained by removing a $\mathrm{PO}_{8}^{+}(2)$ hyperplane. The geometry at infinity of $\mathcal{A S}$ is now the $\mathrm{PO}_{8}^{+}(2)$ polar space with 135 points. As each parallel class of circles in $\mathcal{A S}$ contains 6 circles, we find that the space at infinity of $\Gamma$ consists of all the points of $\mathrm{PO}_{8}^{+}(2)$ polar space at infinity, and of $120 \cdot 63 /(4 \cdot 6)=315$ lines of this polar space. Each of the 120 $\mathrm{PO}_{7}(2)$ hyperplanes of the polar space $\mathcal{A} \mathcal{S}_{\infty}$ meets the space $\Gamma_{\infty}$ at infinity of $\Gamma$ in a subspace isomorphic with $\mathcal{H}$. We will show that $\Gamma_{\infty}$ is isomorphic to the near hexagon associated to the $\mathrm{PSp}_{6}(2)$ dual polar space.

Suppose $p$ is a point and $l$ a line in $\Gamma_{\infty}$, the point $p$ not on $l$. Then there exist $\mathrm{PO}_{7}(2)$ hyperplanes of $\mathcal{A S}_{\infty}$ containing both $p$ and $l$. Such hyperplanes meet $\Gamma$ in a subspace isomorphic to $\mathcal{H}$. So, inside such hyperplanes we see that $l$ contains at least one points at distance $\leq 2$ from $p$ (measured in $\Gamma_{\infty}$ ). If $p$ is collinear (inside $\left.\Gamma_{\infty}\right)$ with two points on $l$, then inside $\mathcal{A S}_{\infty}$ we find $p$ and $l$ to be in a singular plane. However, the intersection of a $\mathrm{PO}_{7}(2)$ hyperplane containing $p$ and $l$ with $\Gamma_{\infty}$ has no plane. This contradiction implies that $p$ is collinear to at most one point on $l$. Suppose $p$ is, inside $\Gamma_{\infty}$, at distance 2 from two points, say $q_{1}$ and $q_{2}$, of $l$. Let $r_{1}$ and $r_{2}$, respectively, be common neighbors of $p$ and $q_{1}$ and $q_{2}$, respectively. 
There is a $\mathrm{PO}_{7}(2)$ hyperplane $H$ of $\mathcal{A} \mathcal{S}_{\infty}$ containing $p, q_{1}, q_{2}, r_{1}$ and $r_{2}$. Intersecting $\Gamma_{\infty}$ with $H$ we obtain a generalized hexagon containing $p, q_{1}, q_{2}, r_{1}$ and $r_{2}$. Inside this hexagon we find that $p$ is collinear to a point on $l$. Thus $\Gamma_{\infty}$ is indeed a near hexagon.

Next we show that any two points of $\Gamma_{\infty}$ at distance 2 are in a quad. Suppose $p$ and $r$ are two such points. Then they are collinear in the polar space $\mathcal{A S}_{\infty}$. But that implies that in any hexagon obtained by intersecting $\Gamma_{\infty}$ with a $\mathrm{PO}_{7}(2)$ hyperplane they stay at distance two and have a common neighbor. By varying these hyperplanes we easily find $p$ and $r$ have at least two common neighbors, from which we deduce with Yanuska's Lemma ([34]), that they are in a quad.

The above shows that $\Gamma_{\infty}$ is a near hexagon with quads and by [8], it is isomorphic to the $\mathrm{PSp}_{6}(2)$ near hexagon. But then $\Gamma$ can be described as the tangent space of the embedded $\mathrm{PSp}_{6}(2)$ near hexagon $\Gamma_{\infty}$ into the $\mathrm{PO}_{8}^{+}(2)$ polar space at infinity of $\mathcal{A S}$. Indeed, $\Gamma$ consists of the points and lines of the affine polar space $\mathcal{A S}$; its circles are those affine planes of $\mathcal{A S}$ whose line at infinity is a line $\Gamma_{\infty}$, which is embedded in the $\mathrm{PO}_{8}^{+}(2)$ hyperplane of $\mathcal{S}$ at infinity of $\mathcal{A S}$. As such an embedding of the $\mathrm{PSp}_{6}(2)$ near hexagon $\Gamma_{\infty}$ is unique up to isomorphism (it is the spin representation of $\left.\mathrm{PSp}_{6}(2)\right)$, we find that $\Gamma$ is unique up to isomorphism. In particular, $\Gamma$ is isomorphic to the extended generalized hexagon on 120 points related to $\mathrm{PSp}_{6}(2)$. (See also [5].)

Finally assume $\mathcal{G}$ to be isomorphic to the graph on 128 points obtained by removing a degenerate hyperplane $\infty^{\perp}$ for some point $\infty$ of the $\operatorname{PSp}_{8}(2)$ polar space. If we fix a point $p$ of $\Gamma$, then the parallel classes of edges and circles of $\mathcal{C}$ on $p$ form the points and lines of a generalized hexagon $H_{p}$ isomorphic to $\mathcal{H}$ embedded into $p^{\perp} \cap \infty^{\perp}$. The lines and singular planes on $\infty$ meeting the generalized hexagon $H_{p}$ in a point respectively line we denote by $\bar{H}_{p}$. This yields an embedding of $\mathcal{H}$ into the $\operatorname{PSp}_{6}(2)$ polar space on lines through $\infty$.

For $p$ and $q$ adjacent points of $\Gamma$ we find that $\bar{H}_{p}$ and $\bar{H}_{q}$ meet in at least 15 lines, as $H_{p}$ and $H_{q}$ meet in 15 lines. For antipodal points $p$ and $q$ in $\Gamma$ (points at distance 3 ) we find $\bar{H}_{p}=\bar{H}_{q}$. But this implies that for any two points $p$ and $q$ of $\Gamma$ the intersection of $\bar{H}_{p}$ and $\bar{H}_{q}$ contains at least 15 lines.

In total, there are 120 different embeddings of $\mathcal{H}$ into a $\mathrm{PSp}_{6}(2)$ polar space. They form an orbit under $\mathrm{PSp}_{6}(2)$. Two distinct embeddings either share 15 lines containing some point at distance $\leq 1$ from a fixed point or 9 non intersecting lines. The graph on these 120 distinct embeddings, with two embeddings adjacent if and only if they intersect in 15 lines is the graph on 120 points of the extended generalized hexagon related to $\operatorname{PSp}_{6}(2)$ consider above. As this graph does not contain 64-cliques, we find that there are points $p$ and $q$ in $\Gamma$ with $\bar{H}_{p}=\bar{H}_{q}$. By switching to an antipodal of $q$, if necessary, we even may assume that these points $p$ and $q$ are adjacent in $\mathcal{G}$. 
Let $r$ be a point different from but cocircular with $p$ and $q$. Let $r_{p}$ and $r_{q}$, respectively, be the point of the $\operatorname{PSp}_{6}(2)$ polar space formed by the line through $\infty$ and the point at infinity of the line through $r$ and $p$ or $q$, respectively. Then $\bar{H}_{r} \cap \bar{H}_{p}$ share at least the 15 lines containing a point at distance $\leq 1$ from $r_{p}$, and $\bar{H}_{r} \cap \bar{H}_{p}$ share at least the 15 lines at distance $\leq 1$ from $r_{q}$. But since $r_{p} \neq r_{q}$ and $\bar{H}_{p}=\bar{H}_{q}$, we find that $\bar{H}_{p}$ and $\bar{H}_{r}$ share more than 15 lines, and therefore are equal. By connectivity we find that for all points $p$ and $q$ of $\Gamma$ we have $\bar{H}_{p}=\bar{H}_{q}$.

As (up to isomorphism) there is only one embedding of $\mathcal{H}$ into a $\mathrm{PSp}_{6}(2)$ polar space, we find that (up to isomorphism) the space $\Gamma_{\infty}$ at infinity of $\Gamma$ is unique. But then, as $\Gamma$ consists of the points and lines of $\mathcal{A S}$ and those affine planes whose line at infinity is in $\Gamma_{\infty}$, uniqueness of $\Gamma$ follows easily. In fact, we find $\Gamma$ to be isomorphic to the extended generalized hexagon on 128 points related to the group $2^{7}: \mathrm{G}_{2}(2)$.

The results of this subsection lead to the following.

Proposition 5.10 Let $\Gamma$ be locally a generalized hexagon of order $(2, t)$. Then $\Gamma$ is isomorphic to the extended generalized hexagon on 128 points related to $2^{7}: \mathrm{G}_{2}(2)$, or the extended generalized hexagon on 120 points related to $\mathrm{PSp}_{6}(2)$.

\subsection{Extensions of the near hexagon of Hamming type}

Now we consider the case that $\Gamma$ is locally the near hexagon of Hamming type, which is the unique near hexagon with parameters $\left(s, t_{2}, t\right)=(2,1,2)$.

Suppose $p$ is a point of $\Gamma$ and let $q$ be a point at distance 2 from $p$ in the graph $\mathcal{G}$. As before, denote by $H$ the geometric hyperplane of $\Gamma_{p}$ consisting of the points not in $p^{\perp} \cap q^{\perp}$. As follows from Lemma 4.7, $\Gamma$ contains a family $\mathcal{E} \mathcal{Q}$ of extended quads. As each point quad pair in the near hexagon of Hamming type is classical, we find that $q^{\perp}$ meets each extended quad on $p$ in 0 or 6 points. Hence $H$ meets each quad of $\Gamma_{p}$ in an ovoid of 3 points or contains the quad. By Proposition 5.1, every point in $H$ is on 0 lines inside $H$, or is deep. Thus if $H$ contains a quad, then all points in this quad are deep. This would imply that all points of the near hexagon are in $H$, which is impossible. Hence $H$ contains no deep points, it is an ovoid and has cardinality 9 . From this we deduce that $\mathcal{G}$ has diameter 2 , and is strongly regular with parameters $(v, k, \lambda, \mu)=(40,27,18,18)$.

Suppose $r$ is a point at distance 2 from $p$ with $p^{\perp} \cap q^{\perp}=p^{\perp} \cap r^{\perp}$, and suppose $u \in p^{\perp} \cap q^{\perp}$. Then in the near hexagon $\Gamma_{u}$ we see that all points at distance at most 2 from $p$ and $q$ are also at distance at most 2 from $r$. One easily checks that this implies that $r=q$. So the 12 points at distance 2 from $p$ all determine different ovoids in $\Gamma_{p}$. But $\Gamma_{p}$ admits exactly 12 ovoids. Indeed, each grid in $\Gamma_{p}$ admits 6 ovoids, and an ovoid of such grid, together with a point outside that grid non collinear with the 3 points of the ovoid in the grid determines a unique ovoid in $\Gamma_{p}$. 
Hence $\Gamma_{p}$ admits $6 \cdot 12 /(9-3)=12$ ovoids. So, each point at distance 2 from $p$ can uniquely be identified with the ovoid it determines.

Fix an ovoid $\mathcal{O}$ of the Hamming near hexagon. Then, inside the Hamming near hexagon, each point of this ovoid is at distance 2 from 6 points inside the ovoid, and at distance 3 from 2 points of the ovoid. There are 9 ovoids of the Hamming near hexagon meeting $\mathcal{O}$ in 3 points (which form an ovoid in a grid of the Hamming near hexagon), and 2 ovoids disjoint from $\mathcal{O}$.

The point $q$ has 9 neighbors in $\mathcal{G}$ that are not adjacent to $p$. These 9 points form an ovoid in $\Gamma_{q}$, and hence each of these points has 6 common neighbors with $q$ which are not in $\Gamma_{p}$. In particular, these 9 points correspond uniquely to the 9 ovoids of $\Gamma_{p}$ meeting $H$ in 3 points. The two points that are at distance 2 from both $p$ and $q$ have 9 common neighbors with $p$ and $q$. So they correspond to the two ovoids in $\Gamma_{p}$ that are disjoint from the ovoid determined by $q$.

It follows from the above, that the graph $\mathcal{G}$ is unique (up to isomorphism). But as the collinearity graph from the near hexagon can be recovered from its distance 1-or-2 graph (collinear points have 13 common neighbors, while points at distance 2 have 11 common neighbors in the distance 1-or-2 graph), $\Gamma$ is also unique. Hence we have proven:

Proposition 5.11 Suppose $\Gamma$ is locally the near hexagon of Hamming type on 27 points, then $\Gamma$ is isomorphic to the extended near hexagon on 40 points related to $\mathrm{PSU}_{4}(2)$.

\subsection{Extensions of the $\mathrm{PSp}_{6}(2)$ near hexagon}

The near hexagon related to the group $\operatorname{PSp}_{6}(2)$ is the unique near hexagon with the parameters $\left(s, t_{2}, t\right)=(2,2,6)$, it has 135 points. Its distance 1-or-2 graph is isomorphic with the graph on the 135 singular points in an orthogonal $\mathrm{PO}_{8}^{+}(2)$ geometry. Two points are adjacent, if and only if they are perpendicular. This graph is a polar graph. Hence, if $\Gamma$ is locally the $\operatorname{PSp}_{6}(2)$ near hexagon, then its collinearity graph is locally a polar graph. In particular, we have:

Lemma 5.12 Suppose $\Gamma$ is locally the $\operatorname{PSp}_{6}(2)$ near hexagon. Then $\mathcal{G}$ is isomorphic to the collinearity graph of the affine polar space obtained by removing a hyperplane from the non degenerate polar space related to $\mathrm{PO}_{10}^{+}(2)$.

Proof. See [15]. ㅁ

The above proposition shows us that $\Gamma$ can be embedded into an affine polar space $\mathcal{A S}$, which together with its space at infinity $\mathcal{A S}_{\infty}$ forms the $\mathrm{PO}_{10}^{+}(2)$ polar 
space $\mathcal{S}$. This polar space $\mathcal{S}$ has two types of hyperplanes; the degenerate hyperplanes with a unique point in the radical, and the $\mathrm{PO}_{9}(2)$ hyperplanes, see [15]. As in Subsection 5.3 we can construct a space $\Gamma_{\infty}$ at infinity for $\Gamma$ by taking all points of the space at infinity of the affine polar space $\mathcal{A S}$ together with those lines at infinity corresponding to parallel classes containing circles in $\mathcal{C}$.

First assume that the removed hyperplane is non degenerate and consider the space $\Gamma_{\infty}$. It contains 255 points. It is embedded in the polar space at infinity $\mathcal{A} \mathcal{S}_{\infty}$ of $\mathcal{A S}$, which is a $\mathrm{PO}_{9}(2)$ polar space. Each point of $\Gamma$ determines a unique $\mathrm{PO}_{8}^{+}(2)$ hyperplane of this polar space meeting the space at infinity of $\Gamma$ in a near hexagon isomorphic to $\Gamma_{p}$. As in Subsection 5.3, it follows from the fact that any point-line pair of the polar space is contained in such a hyperplane that the space at infinity of $\Gamma$ is a near hexagon. By the results of [8], however, we find that no such near hexagon exists.

Thus we can assume that the polar space at infinity of $\mathcal{A S}$ is degenerate. Let $\infty$ be the radical of $\mathcal{A S} \mathcal{S}_{\infty}$. If we fix a point $p$ of $\Gamma$, then the edges and circles on $p$ define a near hexagon $H_{p}$ isomorphic to $\Gamma_{p}$ embedded into the subspace $p^{\perp} \cap \infty^{\perp}$ of the $\mathrm{PO}_{10}^{+}(2)$ polar space. The singular lines and planes of this polar space on the point $\infty$ meeting $H_{p}$ in a point or line, respectively, form a near hexagon embedded in the residue of $\infty$. This embedded near hexagon will be denoted by $\bar{H}_{p}$. We will show that $\bar{H}_{p}$ and $\bar{H}_{q}$ are equal for any two points $p$ and $q$ of $\Gamma$ (compare with Subsection $5.3)$.

There are 240 different embeddings of the $\mathrm{Sp}_{6}(2)$ near hexagon in the $\mathrm{PO}_{8}^{+}(2)$ polar space, forming one orbit under the automorphism group of the polar space. As $\Gamma$ contains 256 points, there are at least two distinct points of $\Gamma, p$ and $q$ say, with $\bar{H}_{p}=\bar{H}_{q}$. For any point $r$ of $\Gamma$ collinear with $p$ and $q$, respectively, we find that $H_{r}$ and $H_{p}$ meet in at least all the points of $H_{p}$ (or $H_{q}$, respectively) not opposite to the point $p r_{\infty}$ (or $q r_{\infty}$ ) which is at infinity of the edge through $r$ and $p$ (or $q$, respectively). But that implies that $\bar{H}_{p}=\bar{H}_{q}$ and $\bar{H}_{r}$ meet in more than the geometric hyperplane of $\bar{H}_{p}$ consisting of all points at distance $\leq 2$ from the point represented by the line through $\infty$ and $p r_{\infty}$. As $\bar{H}_{p}$ is generated by that hyperplane and any point outside it (the complement of the hyperplane is connected, see [6]) we find $\bar{H}_{p}=\bar{H}_{q}$ and $\bar{H}_{r}$ to be the same. By connectivity of $\Gamma_{p}$ we find that $\bar{H}_{r}=\bar{H}_{p}$ for all points $r$ of $\Gamma_{p}$. But then connectivity of $\Gamma$ implies $\bar{H}_{r}=\bar{H}_{p}$ for all $r$ of $\Gamma$. Finally the uniqueness of $\Gamma$ follows easily from the fact that, up to isomorphism, there is a unique embedding of $\Gamma_{p}$ in the $\mathrm{PO}_{8}^{+}(2)$ polar space. This proves:

Proposition 5.13 If $\Gamma$ is an extension of the $\mathrm{PSp}_{6}(2)$ near hexagon, then it is isomorphic to the extension on 256 points related to the group $2^{8}: \mathrm{Sp}_{6}(2)$. 


\subsection{Extensions of the $\mathrm{PSU}_{6}(2)$ near hexagon}

In this subsection we consider the case where for all points $p$ of $\Gamma$ the local near hexagon $\Gamma_{p}$ is isomorphic to the $\mathrm{PSU}_{6}(2)$ near hexagon on 891 points. We prove:

Proposition 5.14 Suppose $\Gamma$ is locally the $\mathrm{PSU}_{6}(2)$ near hexagon, then it is isomorphic to the extension on 2300 points related to $\mathrm{Co}_{2}$.

Suppose $\Gamma$ satisfies the hypothesis of the above proposition. Denote the $\operatorname{PSU}_{6}(2)$ near hexagon by $\mathcal{U}$. Fix a point $p$ of $\Gamma$ and a point $q$ at distance 2 in the collinearity graph of $\Gamma$. By Proposition 5.1 we know that the set $p^{\perp} \cap q^{\perp}$ is the complement of a geometric hyperplane of $\Gamma_{p}$, in which a point is deep or on 15 lines inside the hyperplane.

In the following proposition we classify the geometric hyperplanes of $\mathcal{H}$ having this property.

Proposition 5.15 Let $H$ be a proper geometric hyperplane of $\mathcal{U}$, in which every point is deep or on 15 lines in the hyperplane, then $H$ is one of the orbits of length 567 on the points of $\mathcal{U}$ under a subgroup of $\operatorname{Aut}(\mathcal{U})$ isomorphic to ${ }^{+} \Omega_{6}^{-}(3)$.

Proof. Let $H$ be a hyperplane as in the hypothesis. Suppose $Q$ is a quad in $\mathcal{U}$. Then $H$ contains $Q$ or intersects it in a hyperplane which is either a subquadrangle of order $(2,2)$ in $Q$, or a point and all its neighbors in $Q$.

Let $l$ be a line inside $H$, and suppose $x$ and $y$ are distinct points on this line. There are 5 quads on this line, the intersection of any two of them just being $l$. Since there are at least 15 lines through each of $x$ and $y$ in $H$, there are at most 3 quads on $l$ that are not contained in $H$. Thus any line of $H$ is contained in at least 2 quads inside $H$.

Let $Q$ be a quad of $\mathcal{U}$. Then each point $p$ of $\mathcal{U}$ outside $Q$ is collinear with a unique point $p^{\prime}$ of $Q$. The map $t_{Q}$ which fixes all points of $Q$ and interchanges the two points on each line meeting $Q$ in a point is an automorphism of $\mathcal{U}$. It is an elation of $\mathrm{PSU}_{6}(2)$.

If the quad $Q$ is inside $H$, and $p$ is a point also in $H$, but not in $Q$, then the unique line on $p$ meeting $Q$ is contained in $H$. In particular, $H$ is connected and the involution $t_{Q}$ maps $p$ to a point of $H$. But that implies that $t_{Q}$ stabilizes $H$.

If $p, q$ and $r$ are the points on a line $l$ in $H$, then there is a quad $Q \subseteq H$ on $l$. Inside $Q$ we find a line $m \neq l$ on $r$, which is in a second quad $Q^{\prime}$ distinct from $Q$ but contained in $H$. The involution $t_{Q^{\prime}}$ stabilizes $H$ and maps $p$ to $q$. As $H$ is connected, we find that the stabilizer $G_{H}$ in the group $A u t(\mathcal{U})$ contains the involutions $t_{Q^{\prime}}$, with $Q^{\prime}$ a quad inside $H$, and thus is transitive on $H$. So, $H$ contains no deep points. Moreover, by counting pairs of collinear points $(x, y)$ with $x \in H$ 
and $y \notin H$, we obtain that $12|H|=21 \cdot(891-|H|)$. So, $|H|=567$. Moreover, $G_{H}$ contains $567 x / 27=21 x$ involutions $t_{Q^{\prime}}$, where $x$ is the number of quads in $H$ on each point. As we saw before, this number $x$ is between $15 \cdot 2 / 5=6$ and $15 \cdot 3 / 5=9$. Using the Atlas [17], or with the help of the classification of groups generated by 3 -transpositions, see [21], it is easy to see that $G_{H}$ is isomorphic to ${ }^{+} \Omega_{6}^{-}(3)$. This also follows by the results of [8]. This ends the proof of the proposition.

We notice that the above proposition also follows from results of Pasini and Sphectorov, see [31].

The set of hyperplanes of $\mathcal{U}$ satisfying the hypothesis of the above proposition will be denoted by $\mathcal{G H}$. The group $\operatorname{PSU}_{6}(2)$ contains three conjugacy classes of subgroups isomorphic to ${ }^{+} \Omega_{6}^{-}(3)$ and generated by elations. This implies that the group $\mathrm{PSU}_{6}(2)$ has 3 orbits of length 1408 on the set $\mathcal{G H}$ of geometric hyperplanes satisfying the conditions of the previous proposition. The outer automorphism group of $\mathrm{PSU}_{6}(2)$ induces the full symmetric group $\mathrm{S}_{3}$ on the three classes of subgroups, see [17]. Each of the subgroups isomorphic to ${ }^{+} \Omega_{6}^{-}(3)$ contains a single conjugacy class of 126 elations and acts transitively by conjugation on the remaining $693-126=567$ elations in $\mathrm{PSU}_{6}(2)$.

For each $H \in \mathcal{G H}$ the set of lines in $H$ induces a near hexagon on the 567 points of $H$, the Aschbacher near hexagon, see $[1,8]$. Moreover, we observe that every line of the $\mathrm{PSU}_{6}(2)$ near hexagon is contained in precisely two quads contained in $H$.

In the following lemma, we see how we can distinguish between pairs of geometric hyperplanes in one or in distinct $\operatorname{PSU}_{6}(2)$-orbits on $\mathcal{G H}$.

Lemma 5.16 Let $H$ be a geometric hyperplane in $\mathcal{G H}$. Then there are 567 elements of $\mathcal{G H}$ intersecting $H$ in 375 points, and 840 elements intersecting $H$ in 351 points. Together with $H$, these $1408=1+567+840$ hyperplanes form the $\mathrm{PSU}_{6}(2)$-orbit on $\mathcal{G H}$ containing $H$.

The hyperplane $H$ intersects of each other $\mathrm{PSU}_{6}(2)$-orbit on $\mathcal{G H} 112$ elements in 405 points and 1296 in 357 points.

Proof. Let $G_{H} \simeq^{+} \Omega_{6}^{-}(3)$ be the stabilizer of $H$ in $G=\operatorname{PSU}_{6}(2)$, and let $Q$ be a quad of $\mathcal{U}$ intersecting $H$ in a subquadrangle of order $(2,2)$. Then the geometric hyperplane $H^{t_{Q}}$ intersects $H$ in the 375 points of $H$ that are at distance at most 1 from this subquadrangle. Each hyperplane $H^{\prime}$ intersecting $H$ in the set of points at distance 1 from a subquadrangle of order $(2,2)$ is obtained as the image under an involution $t$ conjugate under $G_{H}$ to $t_{Q}$. As we can find 567 such involutions in $G$, this yields a $G_{H}$-orbit of length 567 . Notice that each point of $H$ is in $567 \cdot 375 / 567=375$ hyperplanes in this $G_{H}$-orbit.

As $\operatorname{PSU}_{6}(2)$ has permutation rank 3 in its action on the cosets of $+\Omega_{6}^{-}(3)$, see [17], the remaining 840 geometric hyperplanes form a $G_{H^{-}}$-orbit. Each point of $\mathcal{H}$ is 
in $896=1408 \cdot 567 / 891$ geometric hyperplanes in the $G$-orbit of $H$. So, since a point of $H$ is in 375 hyperplanes meeting $H$ from the $G_{H}$-orbit described above, such a point is in 520 hyperplanes of the orbit of length 840 . But that implies that each hyperplane of that orbit intersects $H$ in $567 \cdot 520 / 840=351$ points.

By $\mathcal{G} \mathcal{H}_{1}$ we denote the $G$-orbit of $H$. Let $\mathcal{G H}$ with $i=2,3$ be the other two $G$-orbits of length 1408 on $\mathcal{G H}$. From Lemma 2.3 of [30], we know that the stabilizer $G_{H}$ of $H$ has two orbits of length 112 and 1296, respectively, on $\mathcal{G H}_{i}$. If $H^{\prime} \in \mathcal{G H}_{i}$ is an element in the orbit of length 112, then the stabilizer of $H^{\prime}$ is a group isomorphic to $3^{4}: \mathrm{S}_{6}$, see [30]. This group contains 45 elations and $H \cap H^{\prime}$ is the Hall near hexagon as described in [8]. In particular, $H^{\prime} \in \mathcal{G H}$ contains 405 points. Notice that each point of $H$ is in $112 \cdot 405 / 567=80$ hyperplanes in the $G_{H^{-}}$orbit of $H^{\prime}$.

As each point of $H$ is in 896 hyperplanes of $\mathcal{G H}_{i}$, it is in 816 hyperplanes in the $G_{H^{-}}$orbit of length 1296 on $\mathcal{G H}_{i}$. But then by, counting incident point-hyperplane pairs $(p, I)$ with $p \in H$ and $I$ in the $G_{H^{-}}$orbit of length 1296 on $\mathcal{G H}_{i}$, we find that $816 \cdot 567=1296 \cdot|H \cap J|$, where $J$ is an element of the $G_{H^{-}}$orbit of length 1296 . Hence, each such hyperplane $J$ meets $H$ in 357 points. $\square$

Lemma 5.17 The point graph of $\Gamma$ is strongly regular with parameters

$$
(v, k, \lambda, \mu)=(2300,891,378,324) .
$$

Proof. Since the hyperplanes in $\mathcal{G H}$ have no deep points, we see that the graph $\Gamma$ has diameter 2 . The rest is now straightforward by the above.

Lemma 5.18 Let $p$ be a point of $\Gamma$. Then the subgraph of the point graph of $\Gamma$ induced on the vertices distinct and non adjacent to $p$, is a graph which is locally the distance 1-or-2 graph of the Aschbacher near hexagon.

Proof. Straightforward by the above.

Let $p$ be a vertex of $\Gamma$. For each vertex $q$ non adjacent to $p$ denote by $H_{q}$ the geometric hyperplane of the near hexagon induced on $\Gamma_{p}$ consisting of all vertices adjacent to $p$, but not to $q$.

Suppose $q$ and $r$ are two vertices different from $p$ and non adjacent to $p$. Since the two hyperplanes $H_{q}$ and $H_{r}$ can not cover the whole $\Gamma_{p}$, see also 5.16 , there is a vertex $u$ adjacent to $p, q$ and $r$. Inside $\Gamma_{u}$ we find a vertex $w \in H_{q}-H_{r}$. In particular, we find $H_{q}$ and $H_{r}$ to be distinct. Furthermore, we see that there is a vertex adjacent to $q$ and $r$, but not to $p$. In particular, the subgraph of the point graph of $\Gamma$ induced on the set of points at distance 2 from $p$ is connected. 
If $q$ and $r$ are adjacent, then, as can be checked within the Aschbacher near hexagon, they have 246 common neighbors that are not adjacent to $p$, and hence $378-246=132$ common neighbors in $\Gamma_{p}$. Thus $H_{q} \cap H_{r}$ contains $891-2 \cdot 324+132=$ 375 vertices, and $H_{q}$ and $H_{r}$ are in the same $\operatorname{PSU}_{6}(2)$-orbit on $\mathcal{G H}$.

Connectedness of the subgraph of the point graph of $\Gamma$ induced on the set of points at distance 2 from $p$, implies that also when $q$ and $r$ are not adjacent, we find $H_{q}$ and $H_{r}$ in the same $\mathrm{PSU}_{6}(2)$-orbit on $\mathcal{G H}$.

Fix the $\mathrm{PSU}_{6}(2)$-orbits on $\mathcal{G H}$, containing $H_{q}$. (Since Out $\left(\mathrm{PSU}_{6}(2)\right.$ ) is transitive on the three orbits, it is of no concern, which of the three we have to take.) We can identify each of the 1408 points $r$ of $\Gamma$ that are not collinear to $p$ with the unique geometric hyperplane $H_{r}$ in this orbit. On the other hand, for each geometric hyperplane $H$ in this orbit there is a unique vertex $r$ of $\Gamma$ not adjacent to $p$ with $H_{r}=H$. As follows from Lemma 5.16, two vertices $q$ and $r$ of $\Gamma$, not adjacent to $p$ are adjacent, if and only if $\left|H_{q} \cap H_{r}\right|=375$. This shows us that the collinearity graph $\mathcal{G}$ of $\Gamma$ is unique up to isomorphism.

Consider the subgraph of the collinearity graph of $\Gamma$ induced on $\Gamma_{p}$. Then two adjacent vertices of this graph are at distance 1 or 2 in the near hexagon $\Gamma_{p}$. They are at distance 1 if and only if they have 121 common neighbors. Hence, the set $\mathcal{C}$ of circles of $\Gamma$ can uniquely be recovered from the collinearity graph of $\Gamma$, which shows that $\Gamma$ is unique up to isomorphism. We have proved Proposition 5.14.

\subsection{Extensions of the $3^{6}: 2 \cdot \mathrm{M}_{12}$ near hexagon do not exist}

The $3^{6}: 2 \cdot \mathrm{M}_{12}$ near hexagon is the unique example with parameters $\left(s, t_{2}, t\right)=(2,1,11)$. We show that there is no extension of the $3^{6}: 2 . \mathrm{M}_{12}$ near hexagon satisfying condition $(*)$.

Proposition $5.19 \Gamma$ is not an extension of the $3^{6}: 2 \cdot \mathrm{M}_{12}$ near hexagon.

Proof. Suppose $\Gamma$ is locally the $3^{6}: 2 \cdot \mathrm{M}_{12}$ near hexagon, and let $p$ and $q$ be two points of $\Gamma$ at mutual distance 2 in $\mathcal{G}$. By $H$ we denote the geometric hyperplane of $\Gamma_{p}$ consisting of the complement of $p^{\perp} \cap q^{\perp}$ in $\Gamma_{p}$.

From Proposition 5.1 we deduce that each point of $H$ is either deep or on 9 lines inside $H$ and 3 lines meeting the complement of $H$. Counting pairs of collinear points, one in $H$ and the other not, we obtain that $(|H|-|D|) 6=(729-|H|) 12$, where $D$ is the set of deep points in $H$. Hence $|H|=486+|D| / 3 \geq 486$.

Now fix a line $l$ of $\Gamma_{p}$ not in $H$. Let $r$ be the unique point in $l \cap H$. We will count the number of points in $H$ with respect to their distance to $l$.

On $l$ there is a unique point in $H$, the point $r$. Of the points collinear to $r$ there are $18=9 \cdot 2$ inside $H$. The other two points of $l$ are both collinear to 12 points in $H$, one of them being $r$. So at distance 1 from $l$ there are $22+18=40$ points in $H$. 
Now consider a point $u$ at distance 2 from $l$. This point is in a unique grid meeting $l$ in a point. Each point of $l$ is on 55 grids not containing $l$. On $r$, one of these 55 grids meets $H$ in an ovoid, $2 \cdot 9=18$ grids meet $H$ in 5 points of which only 2 are at distance 2 from $r$, and the remaining 36 grids meet $H$ in 5 points or are completely contained in $H$. So, in $H$ there are $2+18 \cdot 2+\alpha \cdot 4=38+4 \alpha$ points at distance 2 from $r$ and $l$, where $\alpha$ equals the number of grids on $r$ completely inside $H$.

Consider a point $x$ on $l$ but not in $H$. On $x$ there are 9 lines which together with $l$ generate a grid meeting $H$ in 5 points. Each of these 9 lines is in 2 grids meeting $H$ in 3 points of an ovoid from which only one point is at distance 2 from $l$. The other two lines on $x$ are on a unique other grid meeting $H$ in an ovoid with just one point at distance 2 from $l$. Thus there are $(9 \cdot 2+2 \cdot 1) / 2=10$ grids on $x$ containing exactly one point of $H$ at distance 2 from $l$ and $55-10=45$ grids on $x$ with 3 points at distance 2 from $l$. So, in $H$ we find $2(10+45 \cdot 3)=290$ points at distance 3 from $r$. In particular, $H$ contains $1+40+38+4 \alpha+290=369+4 \alpha$ points and $|H| \leq 369+36 \cdot 4=513$. Since $486-369=117$ is not divisible by 4 , we find that $H$ has at least one deep point, say $d$. Let $m$ be a line on $d$. We will now count the points with respect to their distance to $m$. If $m$ contains a second deep point, then all grids on $m$ are inside $H$ and we can conclude that the third point of $m$ is also deep. But then we find $3 \cdot 22$ points at distance 1 from $m$ and $\geq 3 \cdot 22 \cdot 14 / 2$ points at distance 2 from $m$. The total number of points in $H$ is then at least $3+66+462$ which contradicts $|H| \leq 513$.

So $d$ is the unique deep point on $m$. This implies that there are $22+16+16=54$ points at distance 1 from $m$ in $H$. On $d$ there are 3 lines generating a grid with $m$ not inside $H$. On each of these three lines we find two grids not containing $m$, nor a point of $H$ at distance 2 from $m$ and 8 grids not on $m$ but contained in $H$. On the 8 lines on $d$ which generate a grid with $m$ contained in $H$ we find 3 grids not containing $m$ nor a point of $H$ at distance 2 from $m$ and 7 grids containing 4 points of $H$ at distance 2 from $m$. Thus inside $H$ we find $(3 \cdot 8+8 \cdot 7) / 2=40$ grids on $d$ each containing 4 points at distance 2 from $l$ and 15 grids not on $m$ having no point of $H$ at distance 2 from $m$. This accounts for 160 points at distance 2 from $l$ and $d$.

Now we consider the grids on a point $e$ of $m$, where $e \neq d$. By arguments similar to the above we find that among the 55 grids on $e$ not containing $m$, there are 12 not containing a point in $H$ and at distance 2 from $m$, there are $24+3$ grids containing 2 points of $H$ at distance 2 from $m$ and finally 16 completely inside $H$. This accounts for $2 \cdot(27 \cdot 2+16 \cdot 4)$ points in $H$ at distance 3 from $d$. In particular, $H$ contains $3+54+160+236=453$ points, which contradicts $|H| \geq 486$. 


\subsection{Extensions of the $\mathrm{M}_{24}$ near hexagon}

In this last subsection we assume $\Gamma$ to be an extension of the $\mathrm{M}_{24}$ near hexagon. The $\mathrm{M}_{24}$ near hexagon is the unique near hexagon with parameters $s=2=t_{2}$ and $t=14$. This near hexagon can be described in terms of the Steiner system $S(24,4,8)$ on a set of 24 symbols. Its points are the octads of this Steiner system, while lines are the partitions of the 24 elements of the Steiner system into three octads. A dodecad is a set of 12 symbols that is the symmetric difference of two octads meeting in 2 symbols. The complement of a dodecad is also a dodecad. To each dodecad we can associate a geometric hyperplane of the near hexagon consisting of those octads that meet the dodecad in precisely 4 symbols. A pair of complementary dodecads is also called a duum. The two dodecads of a duum define the same hyperplane. Using some detailed knowledge of the Steiner system one can verify that two distinct hyperplanes associated to dodecads intersect in 335 or 315 octads, corresponding to the cases where the dodecads intersect in either 4 or 8 points, or 6 points, respectively.

Let $p$ and $q$ be two points at mutual distance 2 in the collinearity graph $\mathcal{G}$ of $\Gamma$, and as usual, let $H_{q}$ denote the geometric hyperplane which is the complement of $p^{\perp} \cap q^{\perp}$ in $\Gamma_{p}$. Then each point in $H$ is on 11 lines inside $H$ and 4 lines meeting $H$ in just one point, or the point is deep.

By the classification of hyperplanes of the $\mathrm{M}_{24}$ near hexagon, see [9], the hyperplane $H_{q}$ is equal to a hyperplane determined by a dodecad as described above. In particular, $H_{q}$ does contain 495 points, none of them being deep. This implies that the collinearity graph of $\Gamma$ has diameter 2 and is strongly regular with parameters $(v, k, \lambda, \mu)=(2048,759,310,264)$. Thus there are 1288 points at distance 2 from $p$. Suppose $r$ is a point not collinear with $p$, but with $p^{\perp} \cap q^{\perp}=p^{\perp} \cap r^{\perp}$, i.e., $H_{q}=H_{r}$. Then for each point $u$ collinear with both $p$ and $q$ we see that in $\Gamma_{u}$ the points at distance at most 2 from $p$ and $q$ are also at distance at most 2 from $r$. Inside $\Gamma_{u}$ we see immediately that this implies that $q$ and $r$ are equal. Hence, the map $q \mapsto H_{q}$ is a bijection between the points at distance 2 from $p$ and the set of 1288 hyperplanes of $\Gamma_{p}$ corresponding to dodecads.

As there are 206 points at distance at most 2 from a fixed point in a hyperplane associated to a dodecad, we find that in the subgraph of $\mathcal{G}$ induced on the set of points at distance two from $p$, two adjacent points have 206 common neighbors. Since any two adjacent points in $\mathcal{G}$ have 310 common neighbors, the two adjacent points $q$ and $r$, both not adjacent to $p$, have $310-206=104$ common neighbors in $p^{\perp}$. In particular, if two points $q$ and $r$, both not adjacent to $p$ are adjacent, then the hyperplanes $H_{q}$ and $H_{r}$ intersect in $335=759-2 \cdot \mu+104$ points. As there are exactly 495 dodecads meeting a fixed dodecad in 4 symbols, we see that two points $q$ and $r$, both not adjacent to $p$ are adjacent, if and only if the dodecads associated to the hyperplanes $H_{q}$ and $H_{r}$ meet in 4 or 8 points. This implies the uniqueness 
of the point graph $\mathcal{G}$ of $\Gamma$. But then the uniqueness of $\Gamma$ follows easily and we have proven:

Proposition 5.20 Let $\Gamma$ be an extension of the $\mathrm{M}_{24}$ near hexagon, then it is isomorphic to the extension on 2048 points related to $2^{11}: \mathrm{M}_{24}$.

\section{Classification of line systems}

In this section we show how Theorem 1.1 follows from Theorem 1.2.

Let $\mathcal{L}$ be a line system as in the hypothesis of Theorem 1.1. Then as already observed in the introduction, this line system carries the structure of an extension of a near hexagon $\Gamma(\mathcal{L})$, the circles of this extended near hexagon being the sets of 4 lines passing through the vertices of a regular tetrahedron centered at the origin. Let $\Sigma$ be the set of norm 3 vectors on the lines in $\mathcal{L}$. Then $\Sigma$ also carries the structure of an extended near hexagon $\Gamma(\Sigma)$, whose circles are now the sets of 4 vertices on the tetrahedra mentioned above. The extended near hexagon $\Gamma(\Sigma)$ is a 2 -fold cover of $\Gamma(\mathcal{L})$, which is an extended near hexagon as in the hypothesis of Theorem 1.3.

We will show that there is a unique way to obtain the 2 -fold cover $\Gamma(\Sigma)$ for each of the extended near hexagons of the conclusion of Theorem 1.3.

Consider $\Gamma(\mathcal{L})$ whose point set is $\mathcal{L}$. For each line $p$ of $\mathcal{L}$ we denote by $+p$ and $-p$ the two norm 3 vectors on $p$. Then $\{ \pm p \mid p \in \mathcal{L}\}$ is the point set of $\Gamma(\Sigma)$. Adjacency in the point graph of $\Gamma(\Sigma)$ is the same has having inner product -1 .

Throughout this section we fix a point $p$ of $\Gamma(\mathcal{L})$ and we show that all adjacencies in $\Gamma(\Sigma)$ can be expressed in terms of adjacencies in $\Gamma(\mathcal{L})$ and subconfigurations of the local near hexagon $\Gamma_{p}$. Without loss of generality, we can assume that all the points adjacent to $+p$ are the points $+q$, and all the points adjacent to $-p$ are the points $-q$, where $q \in p^{\perp}-\{p\}$.

Lemma 6.1 Let $q, r \in p^{\perp}-\{p\}$. Then $+q$ and $+r$, respectively, $-q$ and $-r$ are adjacent, if and only if the points $p, q$ and $r$ are in a circle. The points $+q$ and $-r$ are adjacent, if and only if $q$ and $r$ are adjacent, but not in a circle with $p$.

Proof. Obvious.

Lemma 6.2 Suppose $q$ is a point of $\Gamma(\mathcal{L})$, which is at distance 2 from $p$ in the point graph of $\Gamma(\mathcal{L})$. Then $p^{\perp} \cap q^{\perp}$ is a disjoint union of the sets $\left\{r \in \mathcal{L} \mid+r \in+p^{\perp} \cap+q^{\perp}\right\}$ and $\left\{r \in \mathcal{L} \mid+r \in+p^{\perp} \cap-q^{\perp}\right\}$. Moreover, the sets $+p^{\perp} \cap+q^{\perp}$ and $+p^{\perp} \cap-q^{\perp}$ are both cocliques in the near hexagon $\Gamma(\Sigma)_{+p}$. 
Proof. The two sets $+q^{\perp}$ and $-q^{\perp}$ are disjoint. Thus certainly the two sets $+p^{\perp} \cap$ $+q^{\perp}$ and $+p^{\perp} \cap-q^{\perp}$ are disjoint. If $r \in p^{\perp} \cap q^{\perp}$, then $+r$ is a either adjacent to $+q$ or to $-q$ and hence contained in one of the two sets $+p^{\perp} \cap+q^{\perp}$ or $+p^{\perp} \cap-q^{\perp}$.

Suppose $+r \in+p^{\perp} \cap+q^{\perp}$. Then the points $+q$ and $+p$ are points at distance 3 in the local near hexagon $\Gamma(\Sigma)_{+r}$. In particular, there is no point in $+p^{\perp} \cap+q^{\perp}$ adjacent to $+r$, and we may conclude that $+p^{\perp} \cap+q^{\perp}$ is a coclique. Clearly the same does also hold for $+p^{\perp} \cap-q^{\perp}$.

Lemma 6.3 Let $\Gamma$ be an extended near hexagons of the conclusion of Theorem 1.2. Then, for any pair $p$, $q$ of points at distance two in the point graph of $\Gamma$, there is a unique decomposition of the $\mu$-graph $p^{\perp} \cap q^{\perp}$ into two sets $P_{+}$and $P_{-}$, which are cocliques in both local near hexagons $\Gamma_{p}$ and $\Gamma_{q}$.

Proof. The existence of a decomposition of the $\mu$-graphs into two cocliques follows by the above lemma and the existence of the line systems related to all the extended near hexagons of Theorem 1.2. It remains to show uniqueness.

The $\mu$-graph is the point set of the complement of some hyperplane $H$ of the local near hexagon.

Suppose there are two decompositions $P_{+} \cup P_{-}$and $Q_{+} \cup Q_{-}$of the $\mu$-graph. Then $\left(P_{+} \cap Q_{+}\right) \cup\left(P_{-} \cap Q_{-}\right)$and $\left(P_{+} \cap Q_{-}\right) \cup\left(P_{-} \cap Q_{+}\right)$are joins of different components of the $\mu$-graph. In particular, the $\mu$-graph and hence the complement of the hyperplane $H$ in the local near hexagon is disconnected. We will show that this leads to a contradiction, except in the case where $\Gamma$ is the extended near hexagon related to $\mathrm{PSp}_{6}(2)$.

For generalized quadrangles it is well known that the complements of hyperplanes are connected. Thus we can assume that the diameter of the near hexagon is at least 3 .

Suppose the near hexagon contains quads. Then any two points at distance two are contained in a quad. Thus, if two points are in distinct connected components of the complement of $H$, then they are at distance 3. Suppose $(a, b, c, d)$ is a path of length 3 in the collinearity graph of the near hexagon with $a$ and $d$ not in $H$, and in distinct connected components of the complement of $H$. Thus $b, c \in H$. Let $Q$ be a quad containing $b$ and $c$. As all points of $Q$ that are at distance $\leq 2$ from $a$ and $d$ are in $H$, this quad has to meet $H$ in $b^{\perp} \cap Q$ or in $c^{\perp} \cap Q$, or is contained in $H$. Since there are $t_{2}+2$ lines on $b$ (respectively $c$ ) that are not in $H$, there are $\left(t_{2}+2\right) / t_{2}$ quads on the line through $c$ and $b$ meeting $H$ in $c^{\perp} \cap Q$ (respectively $b^{\perp} \cap Q$ ). But that would imply that the third point of this line is on $2\left(t_{2}+2\right)$ lines not contained in $H$. A contradiction to 5.1.

Now consider the case that $\Gamma$ is locally a generalized hexagon as in $(i v)$ of Theorem 1.2. In this case the $\mu$-graph is the set of points at distance 3 from a fixed 
point in the generalized hexagon $\mathcal{H}$, and as is shown in [16], this graph is connected.

So, for all the extended near hexagon different from the extended generalized hexagon related to $\operatorname{PSp}_{6}(2)$ we have shown the lemma. It remains to consider the case where $\Gamma$ is the extended generalized hexagon related to $\operatorname{PSp}_{6}(2)$. Here the situation is a bit different.

Fix two points $p$ and $q$ as in the hypothesis of the lemma. The $\mu$-graph $M:=$ $p^{\perp} \cap q^{\perp}$ of the collinearity graph of the extended generalized hexagon on 120 points related to $\mathrm{PSp}_{6}(2)$ is the complement of a hyperplane $H$ of the generalized hexagon $\Gamma_{p}$ consisting of 9 lines pairwise at distance 3 in the dual hexagon. Inside the hexagon $\Gamma_{p}$, the complement of such a hyperplane is not connected but has two connected components of 18 points, see [25]. These connected components are distance regular bipartite graph with intersection array $\{3,2,2,1 ; 1,1,2,3\}$. Thus we can partition the 36 vertices in the complement of $H$ into 4 cocliques of 9 vertices, called $C_{i}$, with $i=1, \ldots, 4$, being the 4 halves of the 2 bipartite subgraphs. Up to permutation of the indices we may assume that the two bipartite graphs decomposing $M$ inside $\Gamma_{p}$ have vertex set $C_{1} \cup C_{2}$ and $C_{3} \cup C_{4}$, respectively. Now $C_{1} \cup C_{3}$ and $C_{2} \cup C_{4}$ (or $C_{1} \cup C_{4}$ and $C_{2} \cup C_{3}$ ) yields a partition of the complement of $H$ into two cocliques of $\Gamma_{p}$ of size 18. These are the only two ways to obtain such a partition. We will show that only one of these two partitions satisfies the hypothesis of the lemma. Therefore consider the graph on $M$ induced by $\Gamma_{q}$. At least one of the two partitions obtained inside $\Gamma_{p}$ has to remain a partition into cocliques inside $\Gamma_{q}$. (Indeed, that such a partition exists follows form the existence of the corresponding line system.) Suppose $C_{1} \cup C_{3}$ and $C_{2} \cup C_{4}$ is such a partition. It now suffices to show that $C_{1} \cup C_{4}$ is not a coclique inside $\Gamma_{q}$. Fix a point $c$ of $C_{1}$. Then $c^{\perp} \cap C_{i}$, with $i=2,3,4$ contains 3 points. The three points of $c^{\perp} \cap C_{1}$ are collinear with $c$ inside $\Gamma_{p}$, and hence not inside $\Gamma_{q}$. The three points of $c^{\perp} \cap C_{3}$ as well as the points in $c^{\perp} \cap C_{1}$ are also not collinear to $c$ inside $\Gamma_{q}$ as, by assumption, $C_{1} \cup C_{3}$ is a coclique inside $\Gamma_{q}$. Hence, the three points of $M$ collinear to $c$ inside $\Gamma_{q}$ are the points of $c^{\perp} \cap C_{4}$. In particular, $C_{1} \cup C_{4}$ is not a coclique. This shows that also in this case, there is a unique way to partition $M$ into two sets of size 18 which are both cocliques in $\Gamma_{p}$ and $\Gamma_{q}$.

We are now able to prove that for each of extended near hexagon $\Gamma$ from Theorem 1.2 there exists, up to isomorphism, a unique regular line system $\mathcal{L}$ as in the hypothesis of Theorem 1.1 with $\Gamma(\mathcal{L}) \simeq \Gamma$.

If $q$ is a point of $\Gamma(\mathcal{L})$ at distance 2 from $p$, then the $\mu$-graph $p^{\perp} \cap q^{\perp}$ can be uniquely be partitioned as $P_{+} \cup P_{-}$, as in 6.3. The above lemmas show that without loss of generality we can now assume that the point $+q$ of $\Gamma(\Sigma)$ is the point with $\mu$-graph $+p^{\perp} \cap+q^{\perp}=P_{+}$and $-q$ is the point with $+p^{\perp} \cap-q^{\perp}=P_{-}$.

In the next lemma we will see when two such points are adjacent.

Lemma 6.4 Let $q$ and $r$ be two adjacent points at distance 2 from $p$, and $C=$ 
$\{q, r, u, v\}$ a circle on $q$ and $r$ meeting $p^{\perp}$. Then $+q$ (respectively $\left.+r\right)$ is adjacent to $\epsilon u$ and $\eta v$ for a unique pair $\pi_{q}=(\epsilon, \eta) \in\{+,-\}^{2}$ with $\epsilon \cdot \eta=-$. Moreover, $+q$ and $+r$ are adjacent if and only if $\pi_{q}=\pi_{r}$.

Proof. The point $+q$ is adjacent to $\epsilon u$ and $\eta v$ for some $\epsilon, \eta \in\{+,-\}$. Suppose $+q$ is adjacent to $+u$. The points $+u$ and $+v$ are not adjacent in the near hexagon $\Gamma_{+p}$. Thus $+u$ is adjacent to $-v$. In the near hexagon $\Gamma_{+q}$ there is a unique line through $+u$ and one of the points $+v$ or $-v$. Clearly, this can only be $-v$. Hence $+q$ is also adjacent to $-v$. This proves the first part of the lemma.

The second part follows from the observation that the point $+q$ is adjacent to $+r$ if and only if $+r$ is on the unique line in $\Gamma_{+q}$ through $+u$ and $-v$. Hence if and only if $+r$ is also adjacent to $+u$ and $-v$. $\square$

The above lemmas show that the point graph of $\Gamma(\Sigma)$ is uniquely determined by the isomorphism type of $\Gamma(\mathcal{L})$, which clearly implies that $\Gamma(\Sigma)$ is uniquely determined by $\Gamma(\mathcal{L})$, provided that on each edge at distance 2 from $p$ there is a circle meeting $p^{\perp}$. In other words, provided that the geometric hyperplane which is the complement of the $\mu$-graph in $\Gamma(\mathcal{L})_{p}$ has no deep points. This proves that for each of the extended near hexagons $\Gamma(\mathcal{L})$ of 1.2 there is a unique line system, except possibly, when $\Gamma(\mathcal{L})$ is isomorphic to the extended generalized hexagon related to the group $2^{7}: \mathrm{G}_{2}(2)$. So, assume that $\Gamma(\mathcal{L})$ is isomorphic to this extended generalized hexagon related to the group $2^{7}: \mathrm{G}_{2}(2)$. Then there is a unique point $q$ at distance 3 from $p$ in the point graph of $\Gamma(\mathcal{L})$. There are 126 points $\pm r$ at distance 2 from $+p$, with $r$ at distance 2 from $p$ in the point graph of $\Gamma(\mathcal{L})$. Of these 126 points 63 are adjacent to $+q$, the other 63 to $-q$. The induced subgraphs on the neighbors of $+q$ (or $-q$ ) is a generalized hexagon isomorphic to $\mathcal{H}$. The subgraph induced on the 126 points is isomorphic to the subgraph induced on the 126 points adjacent to $+p$ or $-p$. There is a unique way to partition the 126 points into two sets of 63 points on which the induced subgraph is the point graph of the hexagon $\mathcal{H}$. (Two adjacent vertices are in the same 63 -set if and only if they have 5 common neighbors.) Thus also in this case $\Gamma(\Sigma)$ is uniquely determined by the isomorphism type of $\Gamma(\mathcal{L})$.

It remains to prove that there is a unique presentation of $\Gamma(\Sigma)$ as a set of norm 3 vectors in a line system satisfying the hypothesis of Theorem 1.1. But the point graph of $\Gamma(\Sigma)$ uniquely determines the Gram matrix of the set of vectors $\Sigma$, and hence the line system $\mathcal{L}$. This finishes the proof of Theorem 1.1.

We want to finish the paper with some additional remarks. Consider any of the lines systems $\mathcal{L}$ from Theorem 1.1 different from the one related to $2^{7}: \mathrm{G}_{2}(2)$. Then the point graph of $\Gamma(\mathcal{L})$ is strongly regular. Suppose the parameters of this strongly regular graph are $(k, \lambda, \mu)$ and $l=k(k-\lambda-1) / \mu$. Then the point graph of $\Gamma(\Sigma)$ has distance distribution diagram as follows. 


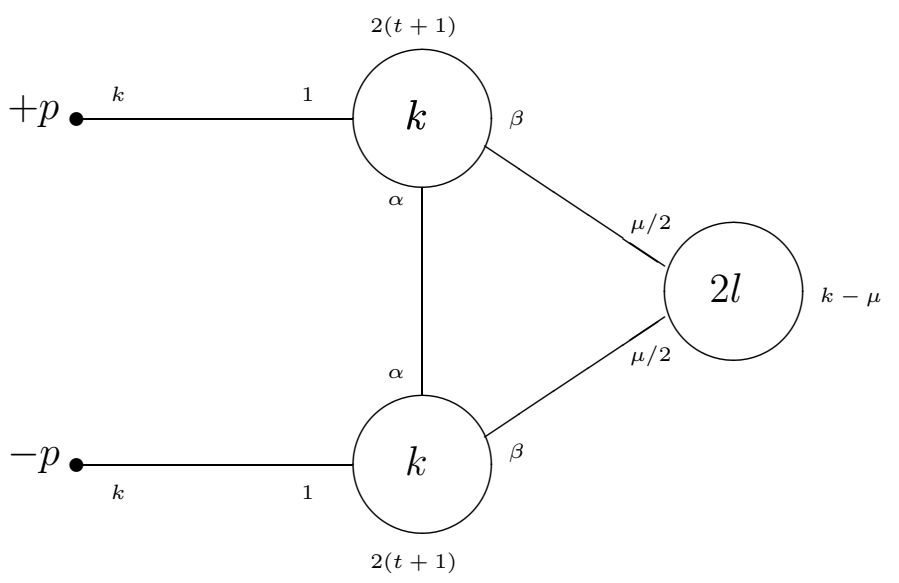

Here $\alpha=\frac{2^{2} t(t+1)}{t_{2}+1}$ and $\beta=\frac{2^{3} t\left(t-t_{2}\right)}{t_{2}+1}$.

If $\Gamma(\mathcal{L})$ is isomorphic to the $2^{7}: \mathrm{G}_{2}(2)$ extended generalized hexagon then the distribution diagram for the point graph of $\Gamma(\Sigma)$ is as follows.

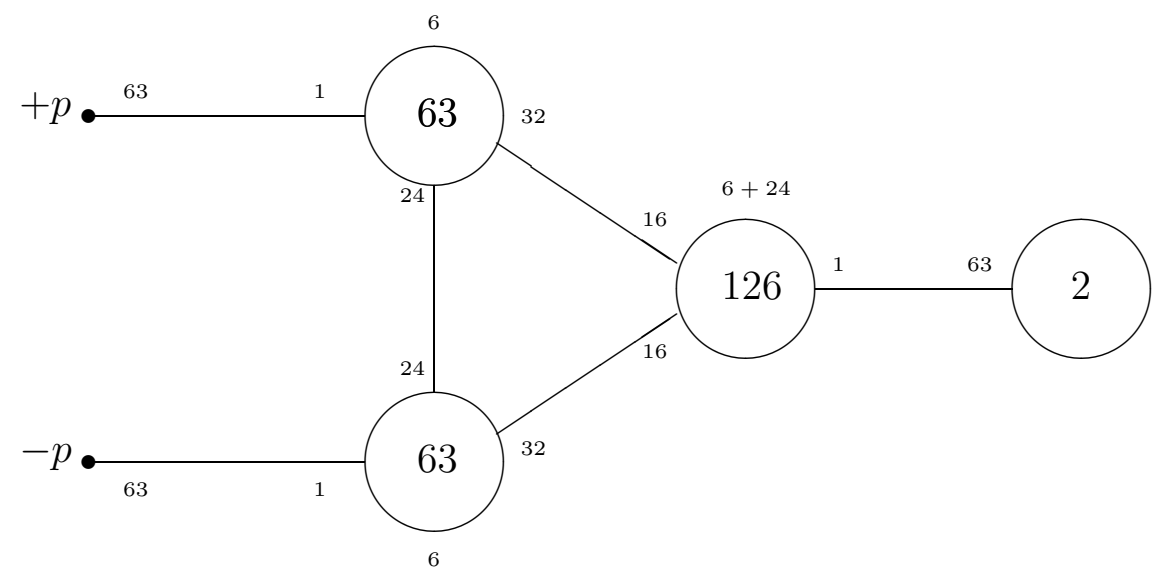

In all cases the line system $\mathcal{L}$ can be realized as follows. Suppose $V$ is the real vector space with basis $\Sigma$ and equipped with an inner product for which $\Sigma$ is an orthogonal basis. Let $A$ be the 0,1 -adjacency matrix of the point graph of $\Gamma(\Sigma)$. From the distribution diagrams it is easy to see that $(+p)-(-p)-(1 / 3)\left(\Sigma_{q \in p^{\perp}-\{p\}}((+q)-\right.$ $(-q)))$ is an eigenvector with eigenvalue $-k / 3$ of $A$, where $k$ is the number of points in the local near hexagon. Orthogonal projection of the vectors of $\Sigma$ onto the $-k / 3$ eigenspace of $A$ and rescaling yields a representation of $\Sigma$ as norm 3 vectors on a tetrahedrally closed regular line system. The dimension of this representation is equal to the multiplicity of the eigenvalue $-k / 3$ of $A$ and equals $|\mathcal{L}| /(1+k / 9)$, see 
$[7]$.

We notice that parts of this section could also be obtained using the theory on covers of diagram geometries, e.g. see [33]. For example, there it is shown that the extended near hexagon on 2300 points related to the group $\mathrm{Co}_{2}$ has a unique universal cover, which is the extended near hexagon related to the 4600 vectors on the 2300 lines in $\mathbb{R}^{23}$ in the line system of Example 3.1.

\section{References}

[1] M. Aschbacher, Flag structures on Tits geometries, Geom. Dedicata 14 (1983), $21-32$.

[2] E. Bannai and N. Sloane, Uniqueness of certain spherical codes, Can. J. Math. 33 (1981), 437-449.

[3] J. van Bon, Some extended generalized hexagons, in Finite geometry and combinatorics, F. De Clerck et al. (eds), LMS Lecture Note Series 191 CUP, Cambridge, 1993, pp. 395-403.

[4] J. van Bon and H. Cuypers, Affine extensions of near hexagons related to the spin module of type $B_{3}$, in: Groups and Geometries, L. di Martino, W.M. Kantor, G. Lunardon, A. Pasini, M.C. Tamburini (eds.), Birkhäuser, Basel, 1998, pp. 215-229.

[5] J. van Bon and H. Cuypers, Affine extensions of generalized polygons, Europ. J. Combinatorics 20 (1999), 571-588.

[6] A.E. Brouwer and R. Blok, The geometry far way from a residue, in: Groups and Geometries, L. di Martino, W.M. Kantor, G. Lunardon, A. Pasini, M.C. Tamburini (eds.), Birkhäuser, Basel, 1998, pp. 29-38.

[7] A.E. Brouwer, A.M. Cohen and A. Neumaier, Distance-regular graphs, Ergebnisse der Math., Springer Verlag, 1989.

[8] A.E. Brouwer, A.M. Cohen, J.I. Hall and H.A. Wilbrink, Near polygons with lines of size three and Fischer spaces, Geom. Dedicata, 49 (1994), 349-368.

[9] A.E. Brouwer, H. Cuypers and E.W. Lambeck, The hyperplanes of the $\mathrm{M}_{24}$ near polygon, to appear in Graphs and Combinatorics.

[10] F. Buekenhout, Diagram geometries for sporadic groups, in Finite groups coming of age, Contemp. Math. 45 (1985), 1-32, AMS, Providence. 
[11] F. Buekenhout and X. Hubaut, Locally polar graphs and related rank 3 groups, J. of Algebra 5 (1977), 391-434.

[12] F. Buekenhout (editor), Handbook of incidence geometry, (1995), NorthHolland, Amsterdam, 1995.

[13] P.J. Cameron, Dual polar spaces, Geom. Dedicata 12 (1982), 75-85.

[14] P.J. Cameron, D. Hughes and A. Pasini, Extended generalised quadrangles, Geom. Dedicata 35 (1990), 193-228.

[15] A.M. Cohen and E.E. Shult, Affine polar spaces, Geom. Dedicata 35 (1990), 43-76.

[16] A.M. Cohen and J. Tits, On generalized hexagons and a near octagon whose lines have three points, Europ. J. of Comb. 6 (1985), 13-27.

[17] J. H. Conway et al., Atlas of finite groups, Clarendon Press, Oxford, 1985.

[18] J. H. Conway and N. Sloane, Sphere packings, lattices and groups, Springer Verlag, Berlin, Heidelberg, New York, 1988.

[19] H. Cuypers, Finite locally generalized quadrangles with affine planes, Europ. J. of Comb. 13 (1992), 439-453.

[20] H. Cuypers and A. Pasini, Locally polar geometries with affine planes, Europ. J. of Comb. 13 (1992), 39-57.

[21] H. Cuypers and J.I. Hall, The 3-transposition groups with trivial center, J. Algebra 178 (1995), 149-193.

[22] H. Cuypers, Extended near hexagons and the Suzuki chain, J. of London Math. Soc.(2) 54 (1996), 1-15.

[23] H. Cuypers, Flag-transitive extensions of buildings of type $\mathrm{G}_{2}$ and $\mathrm{C}_{3}^{*}$, in Groups of Lie type and their geometries, W.M. Kantor and L. di Martino (eds.), LMS Lecture Note Series 207 CUP, Cambridge, 1995, pp. 97-110.

[24] P. Delsarte, J.M. Goethals and J.J. Seidel, Spherical codes and designs, Geom. Dedicata 6 (1977), 363-388.

[25] D. Frohardt and P. Johnson, Geometric hyperplanes in generalized hexagons of order (2,2), Comm. Algebra 22 (1994), 773-797. 


\section{EXTENDED NEAR HEXAGONS AND LINE SYSTEMS}

[26] J.I. Hall and E.E. Shult, Locally cotriangular graphs, Geom. Dedicata 18 (1985), 113-159.

[27] A. Ivanov, D. Pasechnik and S. Sphectorov, Extended $F_{4}$-buildings and the Baby Monster, Invent. Math. 144 (2001) 2, 399-433.

[28] Th. Meixner, A geometric characterization of the simple group $\mathrm{Co}_{2}$, J. of Algebra 165 (1994), 437-445.

[29] A. Neumaier, On norm three vectors in integral euclidean lattices, Math. Z. 183 (1983), 565-574.

[30] D. Pasechnik, Extending polar spaces of rank at least 3, J. Combin. Th. A 72 (1995), 232-242.

[31] A. Pasini and S. Sphectorov, Uniform hyperplanes of finite dual polar spaces of rank 3, J. of Combin. Th. A, 94 (2001), 276-288.

[32] A. Pasini and S. Yoshiara, Flag-transitive Buekenhout geometries, Combinatorics '90 (Gaeta, 1990), Ann. Discrete Math., 52 (1992), 403-447.

[33] M. Ronan, Coverings of certain finite geometries, in Finite geometries and designs, Proc. Sec. Isle of Thorns Conference, 1980, L.M.S. Lecture Note Ser. 49, eds. P. Cameron, J. Hirschfeld and D. Hughes, Cambridge Univ. Press, Cambridge, 1981, pp. 316-331.

[34] E.E. Shult and A. Yanushka, Near polygons and line systems, Geom. Dedicata 9 (1980), 1-72.

[35] H. Van Maldeghem, Generalized Polygons, Birhauser, 1998.

[36] R. Weiss, Extended generalized hexagons, Math. Proc. Camb. Phil. Soc. 108 (1990), 7-19.

[37] R. Weiss, A geometric characterization of the groups $\mathrm{McL}$ and $\mathrm{Co}_{3}$, J. London Math. Soc. 44 (1991), 261-269.

[38] S. Yoshiara, On some extended dual polar spaces I, Algebraic combinatorics (Vladimir, 1991), European J. Combin. 15 (1994), 73-86. 